\title{
Initial-boundary value problems for a reaction-diffusion equation ${ }^{\oplus}$
}

Cite as: J. Math. Phys. 60, 081509 (2019); https://doi.org/10.1063/1.5118767

Submitted: 04 July 2019 . Accepted: 03 August 2019 . Published Online: 27 August 2019

A. Alexandrou Himonas, Dionyssios Mantzavinos, and (Dangchi Yan

COLLECTIONS

EP This paper was selected as an Editor's Pick
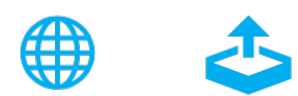

\section{ARTICLES YOU MAY BE INTERESTED IN}

Global well-posedness to some modified 2-D nonhomogeneous Navier-Stokes equations Journal of Mathematical Physics 60, 081510 (2019); https://doi.org/10.1063/1.5123631

Integrable nonlocal Hirota equations

Journal of Mathematical Physics 60, 081508 (2019); https://doi.org/10.1063/1.5013154

The Korteweg-de Vries equation on an interval

Journal of Mathematical Physics 60, 051507 (2019); https://doi.org/10.1063/1.5080366

Journal of

Mathematical Physics

\section{Receive the latest research updates}




\title{
Initial-boundary value problems for a reaction-diffusion equation 라
}

Cite as: J. Math. Phys. 60, 081509 (2019); doi: 10.1063/1.51 18767

Submitted: 4 July 2019 - Accepted: 3 August 2019 •

Published Online: 27 August 2019

\section{A. Alexandrou Himonas, ${ }^{1, a)}$ Dionyssios Mantzavinos, ${ }^{2, b)}$ and Fangchi Yan ${ }^{1,}$}

\author{
AFFILIATIONS \\ 'Department of Mathematics, University of Notre Dame, Notre Dame, Indiana 46556, USA \\ ${ }^{2}$ Department of Mathematics, University of Kansas, Lawrence, Kansas 66045, USA \\ a) E-mail: himonas.1@nd.edu \\ b) Author to whom correspondence should be addressed: mantzavinos@ku.edu \\ ${ }^{c)}$ E-mail: fyan1@nd.edu
}

\begin{abstract}
A novel approach that utilizes Fokas's unified transform is employed for studying a reaction-diffusion equation with power nonlinearity formulated either on the half-line or on a finite interval with data in Sobolev spaces. This approach was recently introduced for initialboundary value problems involving dispersive nonlinear equations such as the nonlinear Schrödinger and the Korteweg-de Vries equations. Thus, the present work extends the new approach from dispersive equations to diffusive ones, demonstrating the universality of the unified transform in the analysis of nonlinear evolution equations on domains with a boundary.
\end{abstract}

Published under license by AIP Publishing. https://doi.org/10.1063/1.5118767

\section{INTRODUCTION}

We consider the following reaction-diffusion equation with power nonlinearity formulated on the half-line with a nonzero Dirichlet boundary condition:

$$
\begin{array}{ll}
u_{t}-u_{x x}=|u|^{p-1} u, & x \in(0, \infty), t \in(0, T), \\
u(x, 0)=u_{0}(x), & x \in[0, \infty), \\
u(0, t)=g_{0}(t), & t \in[0, T],
\end{array}
$$

where $p=2,3,4, \ldots, T<1$, and $u$ is real-valued. For initial and boundary data in appropriate Sobolev spaces, we shall show that the above initial-boundary value problem (IBVP) is well-posed in the sense of Hadamard, i.e., it possesses a unique solution that depends continuously on the data.

The reaction-diffusion equation (1.1a) has been studied extensively and from various points of view, see, for example, Refs. 1, 33, $19,18,4,8,28,5$, and 17 , the books ${ }^{32,23}$ and the references therein. All of these works are concerned either with the initial value problem (IVP) or with IBVPs formulated with zero boundary conditions. In the case of the half-line IBVP (1.1), this corresponds to taking $g_{0} \equiv 0$. On the contrary, here we consider the case of nonzero Dirichlet boundary conditions. In fact, the main objective of this work is to take advantage of a new approach for the well-posedness of nonlinear evolution equations, which relies on the novel solution formulae produced via the unified transform of Fokas ${ }^{9,10}$ for the forced linear counterparts of these equations and which was originally developed for dispersive equations such as the nonlinear Schrödinger, ${ }^{11}$ the Korteweg-de Vries, ${ }^{12}$ and the "good" Boussinesq ${ }^{20}$ equations. That is, the primary purpose of this work is to advance the aforementioned new approach from dispersive equations to diffusive ones and thereby to demonstrate the universality of the unified transform as a tool for studying nonlinear evolution equations on domains with a boundary.

The forced linear counterpart of the nonlinear IBVP (1.1) reads as follows: 


$$
\begin{array}{ll}
u_{t}-u_{x x}=f(x, t), & x \in(0, \infty), t \in(0, T), \\
u(x, 0)=u_{0}(x), & x \in[0, \infty), \\
u(0, t)=g_{0}(t), & t \in[0, T] .
\end{array}
$$

Once a solution formula is derived for the linear problem (1.2), an iteration map can be defined for the solution of the nonlinear problem (1.1). Then, after establishing appropriate estimates on the linear solution formula, it is possible to show well-posedness of the nonlinear problem via a contraction mapping argument. In this connection, we note that, since the boundary datum $g_{0}$ is nonzero, problem (1.2) cannot be solved by converting it into an IVP via the reflection method. Moreover, the well-known sine transform solution formula for this problem is not convenient for the purpose of estimates due to its oscillatory nature. Thus, a significant obstacle is present already at the very beginning of the analysis, namely, at the stage of simply specifying a suitable iteration map. This stands in stark contrast with the case of the IVP, where the linear problem is solved by means of a straightforward application of the Fourier transform.

A novel approach was recently introduced for the study of IBVPs involving nonlinear evolution equations. This approach bypasses the absence of Fourier transform in the IBVP setting by exploiting the unified transform of Fokas for the explicit solution of forced linear evolution IBVPs. ${ }^{9,10}$ In the case of problem (1.2), Fokas's method yields the formula

$$
\begin{aligned}
u(x, t)= & S\left[u_{0}, g_{0} ; f\right](x, t) \\
= & \frac{1}{2 \pi} \int_{k \in \mathbb{R}} e^{i k x-k^{2} t} \widehat{u}_{0}(k) d k-\frac{1}{2 \pi} \int_{k \in \partial D^{+}} e^{i k x-k^{2} t} \widehat{u}_{0}(-k) d k \\
& +\frac{1}{2 \pi} \int_{k \in \mathbb{R}} e^{i k x-k^{2} t} \int_{t^{\prime}=0}^{t} e^{k^{2} t^{\prime}} \widehat{f}\left(k, t^{\prime}\right) d t^{\prime} d k-\frac{1}{2 \pi} \int_{k \in \partial D^{+}} e^{i k x-k^{2} t} \int_{t^{\prime}=0}^{t} e^{k^{2} t^{\prime}} \widehat{f}\left(-k, t^{\prime}\right) d t^{\prime} d k \\
& -\frac{i}{\pi} \int_{k \in \partial D^{+}} e^{i k x-k^{2} t} \widetilde{k g}_{0}\left(k^{2}, T\right) d k,
\end{aligned}
$$

where $\widehat{u}_{0}$ and $\widehat{f}$ are the half-line Fourier transforms of the initial datum $u_{0}$ and the forcing $f$ defined by

$$
\widehat{u}_{0}(k)=\int_{x=0}^{\infty} e^{-i k x} u_{0}(x) d x, \quad \widehat{f}(k, t)=\int_{x=0}^{\infty} e^{-i k x} f(x, t) d x,
$$

the time transform $\widetilde{g}_{0}$ of the boundary datum $g_{0}$ is given by

$$
\widetilde{g}_{0}\left(k^{2}, T\right)=\int_{t=0}^{T} e^{k^{2} t} g_{0}(t) d t
$$

and the contour of integration $\partial D^{+}$is the positively oriented boundary of the region $D^{+}=\{k \in \mathbb{C}: \operatorname{Im}(k) \geqslant|\operatorname{Re}(k)|\}$ of the complex $k$-plane, as shown in Fig. 1.

We shall begin our analysis by employing the unified transform formula (1.3) for studying the forced linear IBVP (1.2) with data in Sobolev spaces $H^{s}$. For a function $f$ on the whole line and $s \geqslant 0$, these spaces are defined in terms of the Fourier transform $\widehat{f}(\xi)=\int_{x \in \mathbb{R}} e^{-i \xi x} f(x) d x$ of $f$ by

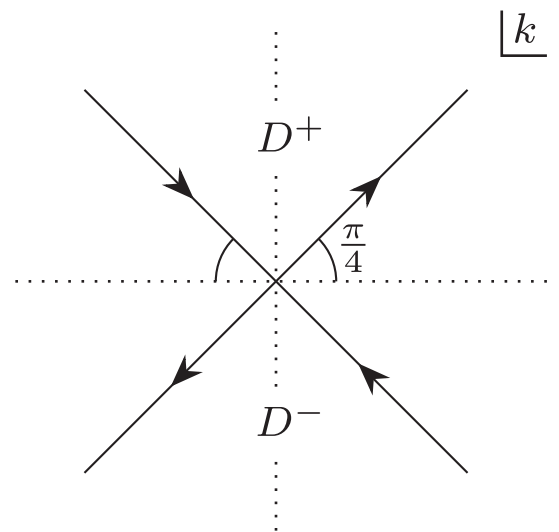

FIG. 1. The regions $D^{ \pm}$with their positively oriented boundaries $\partial D^{ \pm}$ 


$$
H^{s}(\mathbb{R})=\left\{f \in L^{2}(\mathbb{R}):\left(1+\xi^{2}\right)^{\frac{s}{2}} \widehat{f}(\xi) \in L^{2}(\mathbb{R})\right\} .
$$

For functions on an open set $\Omega$ such as the half-line or a finite interval, the relevant Sobolev spaces are obtained as restrictions of their whole-line counterparts according to the definition

$$
H^{s}(\Omega)=\left\{f: f=\left.F\right|_{\Omega} \text { with } F \in H^{s}(\mathbb{R})\right\}, \quad \Omega \subset \mathbb{R} .
$$

Our main result for the forced linear IBVP (1.2) is the following:

Theorem 1.1 (Linear estimates on the half-line). Suppose $\frac{1}{2}<s<\frac{3}{2}, u_{0} \in H_{x}^{s}(0, \infty)$ and $g_{0} \in H_{t}^{(2 s+1) / 4}(0, T)$ with $u_{0}(0)=g_{0}(0)$. Then, the solution $u=S\left[u_{0}, g_{0} ; f\right]$ to the forced linear heat IBVP (1.2) given by the unified transform formula (1.3) admits the space and time estimates,

$$
\begin{array}{r}
\sup _{t \in[0, T]}\|u(t)\|_{H_{x}^{s}(0, \infty)} \leqslant c_{s}\left(\left\|u_{0}\right\|_{H_{x}^{s}(0, \infty)}+\left\|g_{0}\right\|_{H_{t}^{\frac{2 s+1}{4}}(0, T)}+\sqrt{T} \sup _{t \in[0, T]}\|f(t)\|_{H_{x}^{s}(0, \infty)}\right), \\
\sup _{x \in[0, \infty)}\|u(x)\|_{H_{t}^{\frac{2 s+1}{4}}(0, T)} \leqslant c_{s}\left(\left\|u_{0}\right\|_{H_{x}^{s}(0, \infty)}+\left\|g_{0}\right\|_{H_{t}^{\frac{2 s+1}{4}}(0, T)}+\sqrt{T} \sup _{t \in[0, T]}\|f(t)\|_{H_{x}^{s}(0, \infty)}\right),
\end{array}
$$

where $c_{s}=c(s)>0$.

We shall see later that the correspondence $s \leftrightarrow \frac{2 s+1}{4}$ between the regularity (in $x$ ) of $u_{0}$ and the regularity (in $t$ ) of $g_{0}$ stated in Theorem 1.1 emanates from two independent directions: (i) the space regularity of a reduced version of the linear heat IBVP (1.2) with zero initial data and zero forcing and (ii) the time regularity of the linear heat IVP with data in $H_{x}^{s}(\mathbb{R})$. In regard to the latter direction, it is remarkable that the space $H_{t}^{(2 s+1) / 4}(0, T)$ for the time regularity of the linear heat IVP is also associated with the linear Schrödinger IVP (see, for example, Refs. 24 and 11).

The linear estimates of Theorem 1.1 combined with a contraction mapping argument give our next result, which is about the nonlinear $\operatorname{IBVP}(1.1)$.

Theorem 1.2 (Well-posedness on the half-line). Suppose $\frac{1}{2}<s<\frac{3}{2}$ and $\frac{p-1}{2} \in \mathbb{N}$. Then, for $u_{0} \in H_{x}^{s}(0, \infty)$ and $g_{0} \in H_{t}^{(2 s+1) / 4}(0, T)$ with the compatibility condition $u_{0}(0)=g_{0}(0)$, the reaction-diffusion IBVP $(1.1)$ has a unique solution $u \in C\left(\left[0, T^{*}\right] ; H_{x}^{s}(0, \infty)\right)$ which satisfies the estimate

$$
\sup _{t \in\left[0, T^{*}\right]}\|u(t)\|_{H_{x}^{s}(0, \infty)}+\sup _{x \in[0, \infty)}\|u(x)\|_{H_{t}^{\frac{2 s+1}{4}}\left(0, T^{*}\right)} \leqslant 2 c_{s}\left\|\left(u_{0}, g_{0}\right)\right\|_{D}
$$

where $\left\|\left(u_{0}, g_{0}\right)\right\|_{D}=\left\|u_{0}\right\|_{H_{x}^{s}(0, \infty)}+\left\|g_{0}\right\|_{H_{t}^{\frac{2 s+1}{4}}(0, T)}$ and the lifespan $T^{*}$ is given by

$$
T^{*}=\min \left\{T, \frac{1}{p^{2}\left(2 c_{s}\right)^{2 p}\left\|\left(u_{0}, g_{0}\right)\right\|_{D}^{2(p-1)}}\right\}, \quad c_{s}>0 .
$$

Furthermore, the data-to-solution map $\left\{u_{0}, g_{0}\right\} \mapsto u$ is locally Lipschitz continuous.

As noted earlier, the new "unified transform approach to well-posedness" has already been implemented for the nonlinear Schrödinger, the Korteweg-de Vries and the "good" Boussinesq equations on the half-line. ${ }^{11,12,20}$ These three problems share two things in common: (i) they involve dispersive equations and (ii) they are formulated on the half-line. The present work advances the unified transform approach to well-posedness in two different directions: (i) from dispersive to diffusive equations and (ii) from the half-line to a finite interval. The former of these two purposes is accomplished in Theorem 1.2. Concerning the latter one, we note that Theorem 1.1 for the forced linear heat equation on the half-line (1.2) can also be exploited for the following reaction-diffusion IBVP on a finite interval:

$$
\begin{array}{ll}
u_{t}-u_{x x}=|u|^{p-1} u, & x \in(0, \ell), t \in(0, T), \\
u(x, 0)=u_{0}(x), & x \in[0, \ell], \\
u(0, t)=g_{0}(t), u(\ell, t)=h_{0}(t), & t \in[0, T] .
\end{array}
$$

Indeeed, thanks to the linear estimates of Theorem 1.1, it suffices to study the forced linear counterpart of problem (1.10) only in the special case of $f=u_{0}=g_{0}=0$. Then, similarly to the half-line, we prove the following result: 
Theorem 1.3 (Well-posedness on a finite interval). Suppose $\frac{1}{2}<s<\frac{3}{2}$ and $\frac{p-1}{2} \in \mathbb{N}$. Then, for $u_{0} \in H_{x}^{s}(0, \ell), g_{0} \in H_{t}^{(2 s+1) / 4}(0, T)$, and $h_{0} \in H_{t}^{(2 s+1) / 4}(0, T)$ with the compatibility conditions $u_{0}(0)=g_{0}(0)$ and $u_{0}(\ell)=h_{0}(0)$, the reaction-diffusion IBVP $(1.10)$ has a unique solution $u \in C\left(\left[0, T^{*}\right] ; H_{x}^{s}(0, \ell)\right)$ which satisfies the estimate

$$
\sup _{t \in\left[0, T^{*}\right]}\|u(t)\|_{H_{x}^{s}(0, \ell)}+\sup _{x \in[0, \ell]}\|u(x)\|_{H_{t}^{\frac{2 s+1}{4}}\left(0, T^{*}\right)} \leqslant 2 c_{s}\left\|\left(u_{0}, g_{0}, h_{0}\right)\right\|_{D}
$$

where $\left\|\left(u_{0}, g_{0}, h_{0}\right)\right\|_{D}=\left\|u_{0}\right\|_{H_{x}^{s}(0, \ell)}+\left\|g_{0}\right\|_{H_{t}^{\frac{2 s+1}{4}}(0, T)}+\left\|h_{0}\right\|_{H_{t}^{\frac{2 s+1}{4}}(0, T)}$ and

$$
T^{*}=\min \left\{T, \frac{1}{p^{2}\left(2 c_{s}\right)^{2 p}\left\|\left(u_{0}, g_{0}, h_{0}\right)\right\|_{D}^{2(p-1)}}\right\}, \quad c_{s}>0 .
$$

Furthermore, the data-to-solution map $\left\{u_{0}, g_{0}, h_{0}\right\} \mapsto u$ is locally Lipschitz continuous.

We note that apart from the unified transform approach of Refs. 11, 12, and 20, there also exist other approaches in the literature for the well-posedness of dispersive nonlinear IBVPs, namely, the works of Colliander, Kenig, and Holmer ${ }^{6,21,22}$ as well as of Bona, Sun, and Zhang ${ }^{2,3}$ for the Korteweg-de Vries and the nonlinear Schrödinger equations on the half-line. Furthermore, for a different treatment of linear and nonlinear evolution IBVPs that combines the unified transform with inverse scattering techniques, we refer the reader to Fokas and Pelloni, ${ }^{15}$ Pelloni, ${ }^{29,30}$ Fokas, Its, and Sung, ${ }^{13}$ Fokas and Lenells, ${ }^{14,25,26}$ Fokas and Spence, ${ }^{16}$ Deconinck, Pelloni, and Sheils, ${ }^{7}$ Sheils and Smith, ${ }^{31}$ and the references therein.

Structure of the paper. Section II is devoted to the analysis of the linear heat equation on the half-line in the case of zero initial data and zero forcing. This problem is referred to as the pure linear IBVP. The relevant estimates are then combined with those of Sec. III for the linear heat IVP to yield Theorem 1.1 for the forced linear IBVP on the half-line. In turn, this result is employed for carrying out a contraction mapping argument that establishes Theorem 1.2 for the nonlinear IBVP on the half-line (1.1) in the case of "smooth" data ( $\left.s>\frac{1}{2}\right)$. The analog of Theorem 1.1 for the forced linear heat equation on a finite interval is proved in Sec. IV, where Theorem 1.3 for the nonlinear IBVP on a finite interval (1.10) in the case of smooth data is also established. Finally, the case of "rough" data $\left(s<\frac{1}{2}\right)$ both on the half-line and on a finite interval is discussed in Sec. $V$ along with some concluding remarks.

\section{THE PURE LINEAR IBVP ON THE HALF-LINE}

The estimates of Theorem 1.1 for the forced linear problem (1.2) are essential to the contraction mapping argument that leads to the well-posedness of the nonlinear problem (1.1) (Theorem 1.2). Linearity and the superposition principle allow us to treat the IBVP (boundary data) and the IVP (initial data and forcing) components of problem (1.2) separately. We begin with the more challenging IBVP component, which reduces to the following problem that we identify as the pure linear IBVP (see Sec. III for more details on this reduction):

$$
\begin{array}{ll}
v_{t}-v_{x x}=0, & x \in(0, \infty), t \in(0,2), \\
v(x, 0)=0, & x \in[0, \infty), \\
v(0, t)=g(t) \in H_{t}^{\frac{2 s+1}{4}}(\mathbb{R}), & \operatorname{supp}(g) \subset(0,2) .
\end{array}
$$

In the case of the pure linear IBVP (2.1), the unified transform solution formula (1.3) becomes

$$
v(x, t)=S[0, g ; 0](x, t)=\frac{1}{\pi} \int_{k=0}^{\infty} e^{i a^{3} k x+i k^{2} t} k \widehat{g}\left(k^{2}\right) d k+\frac{1}{\pi} \int_{k=0}^{\infty} e^{i a k x-i k^{2} t} k \widehat{g}\left(-k^{2}\right) d k,
$$

where $a=e^{i \frac{\pi}{4}}$ and $\widehat{g}(\tau)=\int_{t \in \mathbb{R}} e^{-i \tau t} g(t) d t$ is the whole-line Fourier transform of $g$ [note that the transform $\widetilde{g}$ defined by (1.5) is equal to $\widehat{g}$ thanks to the compact support of $g$ ]. Using formula (2.2), we shall establish the following estimates for problem (2.1):

Theorem 2.1 (Pure linear IBVP on the half-line). The solution $v=S[0, g ; 0]$ of the pure linear IBVP (2.1) given by the unified transform formula (2.2) admits the space and time estimates,

$$
\begin{gathered}
\sup _{t \in[0,2]}\|v(t)\|_{H_{x}^{s}(0, \infty)} \leqslant c_{s}\|g\|_{H_{t}^{\frac{2 s+1}{4}}(\mathbb{R})}, \quad s \geqslant 0, \\
\sup _{x \in[0, \infty)}\|v(x)\|_{H_{t}^{\frac{2 s+1}{4}}(0,2)} \leqslant c_{s}\|g\|_{H_{t}^{\frac{2 s+1}{4}}(\mathbb{R})}, \quad s \in \mathbb{R} .
\end{gathered}
$$


Remark 2.1. The space estimate (2.3) is the one that motivates the space $H_{t}^{(2 s+1) / 4}(0, T)$ for the boundary data of IBVP (1.1). We shall see later that another, less direct, source of motivation for the boundary data space is the time regularity (3.4) of the linear heat IVP.

Proof of Theorem 2.1. In order to prove the space estimate (2.3), we write $v=v_{1}+v_{2}$ with

$$
\begin{array}{lll}
v_{1}(x, t)=\int_{k=0}^{\infty} e^{i \gamma_{1} k x} G_{1}(k, t) d k, & G_{1}(k, t)=\frac{1}{\pi} e^{i k^{2} t} k \widehat{g}\left(k^{2}\right), & \gamma_{1}=a^{3}=e^{i \frac{3 \pi}{4}}, \\
v_{2}(x, t)=\int_{k=0}^{\infty} e^{i \gamma_{2} k x} G_{2}(k, t) d k, & G_{2}(k, t)=\frac{1}{\pi} e^{-i k^{2} t} k \widehat{g}\left(-k^{2}\right), & \gamma_{2}=a=e^{i \frac{\pi}{4}}
\end{array}
$$

The estimation of $v_{1}$ and $v_{2}$ is entirely analogous. Thus, we only provide the details for $v_{1}$. It is convenient to employ the physical space definition of the $H_{x}^{s}(0, \infty)$-norm,

$$
\left\|v_{1}(t)\right\|_{H_{x}^{s}(0, \infty)}=\sum_{j=0}^{\lfloor s\rfloor}\left\|\partial_{x}^{j} v_{1}(t)\right\|_{L_{x}^{2}(0, \infty)}+\left\|\partial_{x}^{\lfloor s\rfloor} v_{1}(t)\right\|_{\beta}, \quad s=\lfloor s\rfloor+\beta \geqslant 0,0 \leqslant \beta<1
$$

where $\lfloor\cdot\rfloor$ denotes the floor function and, for $0<\beta<1$, the fractional norm $\|\cdot\|_{\beta}$ is defined by

$$
\left\|v_{1}(t)\right\|_{\beta}^{2}=\int_{x=0}^{\infty} \int_{y=0}^{\infty} \frac{\left|v_{1}(x, t)-v_{1}(y, t)\right|^{2}}{|x-y|^{1+2 \beta}} d y d x \simeq \int_{x=0}^{\infty} \int_{z=0}^{\infty} \frac{\left|v_{1}(x+z, t)-v_{1}(x, t)\right|^{2}}{z^{1+2 \beta}} d z d x
$$

There are three cases to consider: (i) $\lfloor s\rfloor=0$ and $\beta \neq 0$; (ii) $\beta=0$; (iii) $\lfloor s\rfloor \neq 0$ and $\beta \neq 0$.

(i) The case $\lfloor s\rfloor=0$ and $\beta \neq 0$. Then, $s=\beta \in(0,1)$ and we need to estimate $\left\|v_{1}(t)\right\|_{L_{x}^{2}(0, \infty)}$ and $\left\|v_{1}(t)\right\|_{\beta}$. The first norm will be estimated together with the $L_{x}^{2}(0, \infty)$-norms of the higher derivatives of $v_{1}$ in case (ii). For the second norm, we have

$$
\left\|v_{1}(t)\right\|_{\beta}^{2} \leqslant \int_{z=0}^{\infty} \int_{x=0}^{\infty} \frac{1}{z^{1+2 \beta}}\left(\int_{k=0}^{\infty}\left|e^{i \gamma_{1} k(x+z)}-e^{i \gamma_{1} k x}\right|\left|G_{1}(k, t)\right| d k\right)^{2} d x d z
$$

and use the following lemma:

Lemma 2.1 (Ref. 12, Lemma 2.1). If $\gamma \in \mathbb{C}$ with $\operatorname{Im}(\gamma)>0$, then

$$
\left|e^{i \gamma k x}-e^{i \gamma k z}\right| \leqslant \sqrt{2}\left(1+\frac{|\operatorname{Re}(\gamma)|}{\operatorname{Im}(\gamma)}\right)\left|e^{-\operatorname{Im}(\gamma) k x}-e^{-\operatorname{Im}(\gamma) k z}\right| \quad \forall k, x, z \geqslant 0 .
$$

Employing Lemma 2.1 with $\gamma=\gamma_{1}=e^{i \frac{3 \pi}{4}}$ and subsequently making the change of variables $\frac{\sqrt{2}}{2} x \rightarrow x, \frac{\sqrt{2}}{2} z \rightarrow z$, we obtain

$$
\left\|v_{1}(t)\right\|_{\beta}^{2} \lesssim \int_{z=0}^{\infty} \frac{1}{z^{1+2 \beta}} \int_{x=0}^{\infty}\left(\int_{k=0}^{\infty} e^{-k x}\left(1-e^{-k z}\right)\left|G_{1}(k, t)\right| d k\right)^{2} d x d z
$$

We identify the $k$-integral in (2.8) as the Laplace transform of $Q_{z, t}(k) \doteq\left(1-e^{-k z}\right)\left|G_{1}(k, t)\right|$. In this connection, we have the following result:

Lemma 2.2 ( $L_{2}$-boundedness of the Laplace transform). The map

$$
\mathcal{L}: \phi \mapsto \int_{\tau=0}^{\infty} e^{-\tau t} \phi(\tau) d \tau
$$

is bounded from $L_{\tau}^{2}(0, \infty)$ into $L_{t}^{2}(0, \infty)$ with

$$
\|\mathcal{L}\{\phi\}\|_{L_{t}^{2}(0, \infty)} \leqslant \sqrt{\pi}\|\phi\|_{L_{\tau}^{2}(0, \infty)} .
$$

A Proof of Lemma 2.2 is available in Ref. 11. Using this lemma for $\phi=Q_{z, t}$, we infer 


$$
\begin{aligned}
\left\|v_{1}(t)\right\|_{\beta}^{2} & \lesssim \int_{z=0}^{\infty} \frac{1}{z^{1+2 \beta}}\left\|Q_{z, t}\right\|_{L_{k}^{2}(0, \infty)}^{2} d z \\
& =\int_{k=0}^{\infty}\left|G_{1}(k, t)\right|^{2} \int_{z=0}^{\infty} \frac{\left(1-e^{-k z}\right)^{2}}{z^{1+2 \beta}} d z d k \lesssim \int_{k=0}^{\infty} k^{2}\left|\widehat{g}\left(k^{2}\right)\right|^{2}|k|^{2 \beta} d k,
\end{aligned}
$$

with the last inequality due to the definition (2.5) of $G_{1}$ and the fact that

$$
\int_{z=0}^{\infty} \frac{\left(1-e^{-k z}\right)^{2}}{z^{1+2 \beta}} d z=k^{2 \beta} \int_{\zeta=0}^{\infty} \frac{\left(1-e^{-\zeta}\right)^{2}}{\zeta^{1+2 \beta}} d \zeta \simeq k^{2 \beta}, \quad 0<\beta<1 .
$$

Then, making the change of variable $\tau=k^{2}$, we obtain

$$
\left\|v_{1}(t)\right\|_{\beta}^{2} \lesssim \int_{\tau=0}^{\infty}|\tau|^{\frac{2 \beta+1}{2}}|\widehat{g}(\tau)|^{2} d \tau \leqslant\|g\|_{H_{t}^{\frac{2 \beta+1}{4}}(\mathbb{R})}, \quad 0<\beta<1,
$$

which is the desired estimate for $v_{1}$ and indicates that the optimal value of $m$ is indeed $\frac{2 s+1}{4}$.

(ii) The case $\beta=0$. Differentiating formula (2.5) and taking its $L^{2}$-norm, we have

$$
\left\|\partial_{x}^{j} v_{1}(t)\right\|_{L_{x}^{2}(0, \infty)} \leqslant\left\|\int_{k=0}^{\infty} e^{-\frac{\sqrt{2}}{2} k x}|k|^{j+1}\left|\widehat{g}\left(k^{2}\right)\right| d k\right\|_{L_{x}^{2}(0, \infty)} .
$$

Therefore, using again Lemma 2.2 for the $L^{2}$-boundedness of the Laplace transform and estimating as in case (i), we find

$$
\left\|\partial_{x}^{j} v_{1}(t)\right\|_{L_{x}^{2}(0, \infty)} \lesssim\left\||k|^{j+1} \widehat{g}\left(k^{2}\right)\right\|_{L_{k}^{2}(0, \infty)} \lesssim\|g\|_{H_{t}^{\frac{2 j+1}{4}}(\mathbb{R})}^{2}, \quad j=0,1, \ldots,\lfloor s\rfloor
$$

so that

$$
\left\|v_{1}(t)\right\|_{H_{x}^{[s]}(0, \infty)} \lesssim\|g\|_{H_{t} \mid \frac{2 l s+1}{4}(\mathbb{R})}, \quad\lfloor s\rfloor \geqslant 0 .
$$

(iii) The case $\lfloor s\rfloor \neq 0$ and $\beta \neq 0$. We now have $s=\lfloor s\rfloor+\beta$ with $\lfloor s\rfloor \geqslant 1$ and $0<\beta<1$. Thanks to our earlier work in cases (i) and (ii), it suffices to estimate the fractional norm $\left\|\partial_{x}^{\lfloor s\rfloor} v_{1}(t)\right\|_{\beta}$. Proceeding as in case (i), we obtain

$$
\left\|\partial_{x}^{\mid s\rfloor} v_{1}(t)\right\|_{\beta}^{2} \lesssim \int_{k=0}^{\infty} k^{2(\lfloor s+\beta)+2}\left|\widehat{g}\left(k^{2}\right)\right|^{2} d k=\left\|k^{\lfloor s\rfloor+\beta+1} \widehat{g}\left(k^{2}\right)\right\|_{L_{k}^{2}(0, \infty)}^{2}
$$

which can be estimated like the corresponding term in case (ii) to yield

$$
\left\|\partial_{x}^{\lfloor s\rfloor} v_{1}(t)\right\|_{\beta} \lesssim\|g\|_{H_{t}^{\frac{2 s+1}{4}}(\mathbb{R})}, \quad\lfloor s\rfloor \geqslant 1 .
$$

Estimates (2.10)-(2.12) combined with the definition (2.7) imply the space estimate (2.3) for $v_{1}$. As noted earlier, this estimate can be established for $v_{2}$ in the exact same way. Hence, the proof of the space estimate (2.3) for $v$ is complete.

The time estimate (2.4) is easier to obtain. In particular, the change of variable $k=\sqrt{\tau}$ turns formula (2.2) into

$$
v(x, t) \simeq \int_{\tau=0}^{\infty} e^{i a^{3} \sqrt{\tau} x+i \tau t} \widehat{g}(\tau) d \tau+\int_{\tau=-\infty}^{0} e^{i a \sqrt{-\tau} x+i \tau t} \widehat{g}(\tau) d \tau .
$$

Therefore, since the $x$-exponentials involved above are bounded by 1 for $x \geqslant 0$, we find

$$
\begin{aligned}
\|v(x)\|_{H_{t}^{2}+\frac{2 s+1}{4}(\mathbb{R})} & \lesssim \int_{\tau=0}^{\infty}\left(1+\tau^{2}\right)^{\frac{2 s+1}{4}} e^{-\sqrt{2} \sqrt{\tau} x}|\widehat{g}(\tau)|^{2} d \tau+\int_{\tau=-\infty}^{0}\left(1+\tau^{2}\right)^{\frac{2 s+1}{4}} e^{-\sqrt{2} \sqrt{-\tau} x}|\widehat{g}(\tau)|^{2} d \tau \\
& \leqslant \int_{\tau=0}^{\infty}\left(1+\tau^{2}\right)^{\frac{2 s+1}{4}}|\widehat{g}(\tau)|^{2} d \tau+\int_{\tau=-\infty}^{0}\left(1+\tau^{2}\right)^{\frac{2 s+1}{4}}|\widehat{g}(\tau)|^{2} d \tau=\|g\|_{H_{t}^{2}}^{2}(\mathbb{R})
\end{aligned}
$$

The Proof of Theorem 2.1 is complete. 


\section{LINEAR IVP ESTIMATES AND PROOF OF THEOREMS 1.1 AND 1.2}

In this section, we analyze the IVP component of the forced linear IBVP (1.1). Then, combining the relevant estimates with those of Theorem 2.1 for the pure linear IBVP, we deduce the linear Theorem 1.1. Finally, we combine this result with a contraction mapping argument in order to infer the nonlinear Theorem 1.2.

We begin with the homogeneous linear heat IVP

$$
\begin{aligned}
& U_{t}-U_{x x}=0, \quad x \in \mathbb{R}, t \in(0, T), \\
& U(x, 0)=U_{0}(x) \in H_{x}^{s}(\mathbb{R}),
\end{aligned}
$$

which can be solved via the whole-line Fourier transform $\widehat{U}(\xi, t)=\int_{x \in \mathbb{R}} e^{-i \xi x} U(x, t) d x$ to yield

$$
U(x, t)=S\left[U_{0} ; 0\right](x, t)=\frac{1}{2 \pi} \int_{\xi \in \mathbb{R}} e^{i \xi x-\xi^{2} t} \widehat{U}_{0}(\xi) d \xi .
$$

Theorem 3.1 (Estimates for the homogeneous linear IVP). The solution $U=S\left[U_{0} ; 0\right]$ of the linear heat IVP (3.1) given by formula (3.2) admits the estimates

$$
\begin{array}{cc}
\sup _{t \in[0, T]}\|U(t)\|_{H_{x}^{s}(\mathbb{R})} \leqslant\left\|U_{0}\right\|_{H_{x}^{s}(\mathbb{R}),}, & s \in \mathbb{R}, \\
\sup _{x \in \mathbb{R}}\|U(x)\|_{H_{t}^{\frac{2 s+1}{4}}(0, T)} \leqslant c_{s}\left\|U_{0}\right\|_{H_{x}^{s}(\mathbb{R})}, & -\frac{1}{2} \leqslant s<\frac{3}{2} .
\end{array}
$$

Remark 3.1. The time estimate (3.4) provides a second source of motivation [in addition to the pure linear IBVP estimate (2.3)] for the boundary data space of the half-line problem (1.1). It is interesting to note that, despite the diffusive nature of the heat equation, the Sobolev exponent $\frac{2 s+1}{4}$ is precisely the one appearing in the corresponding estimate for the (dispersive) linear Schrödinger equation (see Refs. 24 and 11).

Proof of Theorem 3.1. The solution formula (3.2) combined with the definition of the $H_{x}^{s}(\mathbb{R})$-norm implies the space estimate (3.3) for all $s \in \mathbb{R}$ and all $t \geqslant 0$,

$$
\|U(t)\|_{H_{x}^{s}(\mathbb{R})}^{2}=\int_{\xi \in \mathbb{R}}\left(1+\xi^{2}\right)^{s}\left|e^{-\xi^{2} t} \widehat{U}_{0}(\xi)\right|^{2} d \xi \leqslant \int_{\xi \in \mathbb{R}}\left(1+\xi^{2}\right)^{s}\left|\widehat{U}_{0}(\xi)\right|^{2} d \xi=\left\|U_{0}\right\|_{H_{x}^{s}(\mathbb{R})}^{2}
$$

The proof of the time estimate (3.4) is more involved. Letting $m=\frac{2 s+1}{4}$ and noting that $-\frac{1}{2} \leqslant s<\frac{3}{2}$ corresponds to $0 \leqslant m<1$, we employ definition (2.7) to write

$$
\|U(x)\|_{H_{t}^{m}(0, T)}=\|U(x)\|_{L_{t}^{2}(0, T)}+\|U(x)\|_{m}, \quad 0 \leqslant m<1,
$$

where for $0<m<1$, the fractional norm $\|\cdot\|_{m}$ is defined by

$$
\|U(x)\|_{m}^{2}=\int_{t=0}^{T} \int_{t^{\prime}=0}^{T} \frac{\left|U(x, t)-U\left(x, t^{\prime}\right)\right|^{2}}{\left|t-t^{\prime}\right|^{1+2 m}} d t^{\prime} d t \simeq \int_{t=0}^{T} \int_{z=0}^{T-t} \frac{|U(x, t+z)-U(x, t)|^{2}}{z^{1+2 m}} d z d t .
$$

For the norm $\|U(x)\|_{L_{t}^{2}(0, T)}$, we have

$$
\begin{aligned}
\|U(x)\|_{L_{t}^{2}(0, T)} \lesssim & \int_{t=0}^{T}\left[\int_{\xi=0}^{1} e^{-\xi^{2} t}\left(\left|\widehat{U}_{0}(-\xi)\right|+\left|\widehat{U}_{0}(\xi)\right|\right) d \xi\right]^{2} d t \\
& +\int_{t=0}^{T}\left[\int_{\xi=1}^{\infty} e^{-\xi^{2} t}\left(\left|\widehat{U}_{0}(-\xi)\right|+\left|\widehat{U}_{0}(\xi)\right|\right) d \xi\right]^{2} d t .
\end{aligned}
$$

By the Cauchy-Schwarz inequality, we find

$$
(3.6 a) \lesssim \int_{t=0}^{T}\left(\int_{\xi=0}^{1} e^{-2 \xi^{2} t}\left(1+\xi^{2}\right)^{-s} d \xi\right)\left\|U_{0}\right\|_{H_{x}^{s}(\mathbb{R})}^{2} d t \leqslant c_{s} T\left\|U_{0}\right\|_{H_{x}^{s}(\mathbb{R})}^{2}, \quad s \in \mathbb{R} .
$$


Moreover, making the change of variable $\xi=\sqrt{\tau}$, we have

$$
(3.6 b)=\int_{t=0}^{T}\left(\int_{\tau=1}^{\infty} e^{-\tau t} \frac{\left|\widehat{U}_{0}(-\sqrt{\tau})\right|+\left|\widehat{U}_{0}(\sqrt{\tau})\right|}{2 \sqrt{\tau}} d \tau\right)^{2} d t \lesssim\|\mathcal{L}\{\phi\}\|_{L_{t}^{2}(0, \infty)}^{2}
$$

where $\mathcal{L}\{\phi\}(t)=\int_{\tau=0}^{\infty} e^{-\tau t} \phi(\tau) d \tau$ is the Laplace transform of the function

$$
\phi(\tau)= \begin{cases}\tau^{-\frac{1}{2}}\left(\left|\widehat{U}_{0}(-\sqrt{\tau})\right|+\left|\widehat{U}_{0}(\sqrt{\tau})\right|\right), & \tau \geqslant 1, \\ 0, & 0 \leqslant \tau<1 .\end{cases}
$$

Hence, Lemma 2.2 yields

$$
(3.6 b) \lesssim \int_{\tau=1}^{\infty} \tau^{-1}\left|\widehat{U}_{0}(-\sqrt{\tau})\right|^{2} d \tau+\int_{\tau=1}^{\infty} \tau^{-1}\left|\widehat{U}_{0}(\sqrt{\tau})\right|^{2} d \tau
$$

and, letting $\xi=-\sqrt{\tau}$ and $\xi=\sqrt{\tau}$ in the first and the second integral, respectively, we obtain

$$
(3.6 b) \lesssim \int_{|\xi| \geqslant 1}|\xi|^{-1}\left|\widehat{U}_{0}(\xi)\right|^{2} d \xi \leqslant\left\|U_{0}\right\|_{H_{x}^{-\frac{1}{2}}(\mathbb{R})}^{2} \leqslant\left\|U_{0}\right\|_{H_{x}^{s}(\mathbb{R})}^{2}, \quad s \geqslant-\frac{1}{2}
$$

Combining estimates (3.7) and (3.9), we find

$$
\|U(x)\|_{L_{t}^{2}(0, T)} \lesssim\left\|U_{0}\right\|_{H_{x}^{s}(\mathbb{R})}, \quad s \geqslant-\frac{1}{2}, x \in \mathbb{R} .
$$

For the fractional norm $\|U(x)\|_{m}$, starting from formula (3.2), we compute

$$
\begin{aligned}
|U(x, t+z)-U(x, t)| \lesssim & \int_{\xi=0}^{1} e^{-\xi^{2} t}\left(1-e^{-\xi^{2} z}\right)\left(\left|\widehat{U}_{0}(-\xi)\right|+\left|\widehat{U}_{0}(\xi)\right|\right) d \xi \\
& +\int_{\xi=1}^{\infty} e^{-\xi^{2} t}\left(1-e^{-\xi^{2} z}\right)\left(\left|\widehat{U}_{0}(-\xi)\right|+\left|\widehat{U}_{0}(\xi)\right|\right) d \xi
\end{aligned}
$$

Hence, from the definition of $\|U(x)\|_{m}$, we have

$$
\begin{aligned}
\|U(x)\|_{m}^{2} \lesssim & \int_{t=0}^{T} \int_{z=0}^{T-t} \frac{1}{z^{1+2 m}}\left[\int_{\xi=0}^{1} e^{-\xi^{2} t}\left(1-e^{-\xi^{2} z}\right)\left(\left|\widehat{U}_{0}(-\xi)\right|+\left|\widehat{U}_{0}(\xi)\right|\right) d \xi\right]^{2} d z d t \\
& +\int_{t=0}^{T} \int_{z=0}^{T-t} \frac{1}{z^{1+2 m}}\left[\int_{\xi=1}^{\infty} e^{-\xi^{2} t}\left(1-e^{-\xi^{2} z}\right)\left(\left|\widehat{U}_{0}(-\xi)\right|+\left|\widehat{U}_{0}(\xi)\right|\right) d \xi\right]^{2} d z d t .
\end{aligned}
$$

For any $s \in \mathbb{R}$, the Cauchy-Schwarz inequality implies

$$
(3.11 a) \lesssim T\left\|U_{0}\right\|_{H_{x}^{s}(\mathbb{R})}^{2} \int_{\xi=0}^{1}\left(1+\xi^{2}\right)^{-s} \int_{z=0}^{T} \frac{\left(1-e^{-\xi^{2} z}\right)^{2}}{z^{1+2 m}} d z d \xi .
$$

Therefore, employing (2.9) for $k=\xi^{2}$ and $\beta=m$, we find

$$
(3.11 a) \lesssim T\left\|U_{0}\right\|_{H_{x}^{s}(\mathbb{R})}^{2} \int_{\xi=0}^{1}\left(1+\xi^{2}\right)^{-s} \xi^{4 m} d \xi=c_{s} T\left\|U_{0}\right\|_{H_{x}^{s}(\mathbb{R})}^{2}, \quad-\frac{1}{2}<s<\frac{3}{2} .
$$

Furthermore, letting $\xi=\sqrt{\tau}$, we have

$$
(3.11 b) \lesssim \int_{z=0}^{T} \frac{1}{z^{1+2 m}}\|\mathcal{L}\{\phi\}(z)\|_{L_{t}^{2}(0, \infty)}^{2} d z,
$$

where 


$$
\phi(\tau)= \begin{cases}\tau^{-\frac{1}{2}}\left(1-e^{-\tau z}\right)\left(\left|\widehat{U}_{0}(-\sqrt{\tau})\right|+\left|\widehat{U}_{0}(\sqrt{\tau})\right|\right), & \tau \geqslant 1, \\ 0, & 0 \leqslant \tau<1 .\end{cases}
$$

Thus, by the Laplace transform bound of Lemma 2.2, we find

$$
(3.11 b) \lesssim \int_{\xi=1}^{\infty} \xi^{-1}\left(\left|\widehat{U}_{0}(-\xi)\right|+\left|\widehat{U}_{0}(\xi)\right|\right)^{2} \int_{z=0}^{T} \frac{\left(1-e^{-\xi^{2} z}\right)^{2}}{z^{1+2 m}} d z d \xi
$$

and, using (2.9) as before, we obtain

$$
(3.11 b) \lesssim \int_{\xi=1}^{\infty} \xi^{4 m-1}\left(\left|\widehat{U}_{0}(-\xi)\right|+\left|\widehat{U}_{0}(\xi)\right|\right)^{2} d \xi \lesssim\left\|U_{0}\right\|_{H_{x}^{s}(\mathbb{R})}^{2}, \quad-\frac{1}{2}<s<\frac{3}{2} .
$$

Combining estimates (3.12) and (3.13), we deduce

$$
\|U(x)\|_{m} \lesssim\left\|U_{0}\right\|_{H_{x}^{s}(\mathbb{R})}, \quad-\frac{1}{2}<s<\frac{3}{2}, x \in \mathbb{R},
$$

which together with estimate (3.10) and the definition (3.5) yield estimate (3.4).

After analyzing the homogeneous linear IVP (3.1), it remains to estimate the following forced linear IVP with zero forcing:

$$
\begin{array}{ll}
W_{t}-W_{x x}=F(x, t), & x \in \mathbb{R}, t \in(0, T), \\
W(x, 0)=0, & x \in \mathbb{R},
\end{array}
$$

whose solution via the whole-line Fourier transform $\widehat{W}(\xi, t)=\int_{x \in \mathbb{R}} e^{-i \xi x} W(x, t) d x$ is given by

$$
\begin{aligned}
W(x, t)=S[0 ; F](x, t) & =\frac{1}{2 \pi} \int_{\xi \in \mathbb{R}} \int_{t^{\prime}=0}^{t} e^{i \xi x-\xi^{2}\left(t-t^{\prime}\right)} \widehat{F}\left(\xi, t^{\prime}\right) d t^{\prime} d \xi \\
& =\int_{t^{\prime}=0}^{t} S\left[F\left(\cdot, t^{\prime}\right) ; 0\right]\left(x, t-t^{\prime}\right) d t^{\prime}
\end{aligned}
$$

Theorem 3.2 (Estimates for the forced linear IVP). The solution $W=S[0 ; F]$ of the forced linear heat IVP (3.15) given by (3.16) admits the space and time estimates,

$$
\begin{aligned}
\sup _{t \in[0, T]}\|W(t)\|_{H_{x}^{s}(\mathbb{R})} \leqslant T \sup _{t \in[0, T]}\|F(t)\|_{H_{x}^{s}(\mathbb{R}),} & s \in \mathbb{R}, \\
\sup _{x \in \mathbb{R}}\|W(x)\|_{H_{t}^{\frac{2 s+1}{4}}(0, T)} \leqslant c_{s} \sqrt{T} \sup _{t \in[0, T]}\|F(t)\|_{H_{x}^{s}(\mathbb{R}),} & \frac{1}{2}<s<\frac{3}{2} .
\end{aligned}
$$

Proof of Theorem 3.2. The space estimate (3.17) follows by combining the Duhamel formula (3.16b) with Minkowski's integral inequality and the homogeneous space estimate (3.3).

Concerning the time estimate (3.18), we set $m=\frac{2 s+1}{4}$ and employ the physical space Sobolev norm (3.5). Since $\frac{1}{2}<s<\frac{3}{2}$ corresponds to $\frac{1}{2}<m<1$, we only need to estimate $\|W(x)\|_{L_{t}^{2}(0, T)}$ and $\|W(x)\|_{m}$. For the first term, starting from the Duhamel representation (3.16b) and combining Minkowski's integral inequality with the homogeneous time estimate (3.4), we find

$$
\|W(x)\|_{L_{t}^{2}(0, T)} \leqslant \int_{t^{\prime}=0}^{T}\left\|S\left[F\left(\cdot, t^{\prime}\right) ; 0\right]\left(x, t-t^{\prime}\right)\right\|_{L_{t}^{2}(0, T)} d t^{\prime} \leqslant T \sup _{t \in[0, T]}\|F(t)\|_{H_{x}^{s}(\mathbb{R})} .
$$

For the second term, writing

$$
\begin{aligned}
W(x, t+z)-W(x, t)= & \int_{t^{\prime}=0}^{t}\left[S\left[F\left(\cdot, t^{\prime}\right) ; 0\right]\left(x, t+z-t^{\prime}\right)-S\left[F\left(\cdot, t^{\prime}\right) ; 0\right]\left(x, t-t^{\prime}\right)\right] d t^{\prime} \\
& +\int_{t^{\prime}=t}^{t+z} S\left[F\left(\cdot, t^{\prime}\right) ; 0\right]\left(x, t+z-t^{\prime}\right) d t^{\prime}
\end{aligned}
$$


and recalling the definition of the fractional norm, we have

$$
\begin{aligned}
\|W(x)\|_{m}^{2} \lesssim & \int_{t=0}^{T} \int_{z=0}^{T-t} \frac{1}{z^{1+2 m}}\left|\int_{t^{\prime}=0}^{t}\left[S\left[F\left(\cdot, t^{\prime}\right) ; 0\right]\left(x, t+z-t^{\prime}\right)-S\left[F\left(\cdot, t^{\prime}\right) ; 0\right]\left(x, t-t^{\prime}\right)\right] d t^{\prime}\right|^{2} d z d t \\
& +\int_{t=0}^{T} \int_{z=0}^{T-t} \frac{1}{z^{1+2 m}}\left|\int_{t^{\prime}=t}^{t+z} S\left[F\left(\cdot, t^{\prime}\right) ; 0\right]\left(x, t+z-t^{\prime}\right) d t^{\prime}\right|^{2} d z d t .
\end{aligned}
$$

By Minkowski’s integral inequality and estimate (3.4), we obtain

$$
(3.20 a) \leqslant\left(\int_{t^{\prime}=0}^{T}\left\|S\left[F\left(\cdot, t^{\prime}\right) ; 0\right]\left(x, t-t^{\prime}\right)\right\|_{m} d t^{\prime}\right)^{2} \lesssim\left(T \sup _{t \in[0, T]}\|F(t)\|_{H_{x}^{s}(\mathbb{R})}\right)^{2} .
$$

Furthermore, using the representation (3.16a), we have

$$
(3.20 b) \leqslant \int_{t=0}^{T} \int_{z=0}^{T-t} \frac{1}{z^{1+2 m}}\left\|\frac{1}{2 \pi} \int_{\xi \in \mathbb{R}} e^{i \xi x} \int_{t^{\prime}=t}^{t+z} e^{-\xi^{2}\left(t+z-t^{\prime}\right)} \widehat{F}\left(\xi, t^{\prime}\right) d t^{\prime} d \xi\right\|_{L_{x}^{\infty}(\mathbb{R})}^{2} d z d t .
$$

Hence, the Sobolev embedding theorem for $s>\frac{1}{2}$ implies

$$
\begin{aligned}
(3.20 b) & \leqslant \int_{t=0}^{T} \int_{z=0}^{T-t} \frac{1}{z^{1+2 m}}\left\|\frac{1}{2 \pi} \int_{\xi \in \mathbb{R}} e^{i \xi x} \int_{t^{\prime}=t}^{t+z} e^{-\xi^{2}\left(t+z-t^{\prime}\right)} \widehat{F}\left(\xi, t^{\prime}\right) d t^{\prime} d \xi\right\|_{H_{x}^{s}(\mathbb{R})}^{2} d z d t \\
& \leqslant \int_{t=0}^{T} \int_{z=0}^{T-t} \frac{1}{z^{1+2 m}} \int_{\xi \in \mathbb{R}}\left(1+\xi^{2}\right)^{s}\left(\int_{t^{\prime}=t}^{t+z}\left|\widehat{F}\left(\xi, t^{\prime}\right)\right| d t^{\prime}\right)^{2} d \xi d z d t,
\end{aligned}
$$

and Minkowski's integral inequality further yields

$$
(3.20 b) \leqslant \int_{t=0}^{T} \int_{z=0}^{T-t} \sup _{t^{\prime} \in[t, t+z]}\left\|F\left(t^{\prime}\right)\right\|_{H_{x}^{s}(\mathbb{R})}^{2} \cdot z^{1-2 m} d z d t \lesssim T^{3-2 m} \sup _{t \in[0, T]}\|F(t)\|_{H_{x}^{s}(\mathbb{R})}^{2},
$$

after recalling that $m<1$. Combining (3.20)-(3.22) and the fact that $T<1$, we deduce

$$
\|W(x)\|_{m \lesssim} \sqrt{T} \sup _{t \in[0, T]}\|F(t)\|_{H_{x}^{s}(\mathbb{R})} .
$$

Overall, estimates (3.19) and (3.23) together with the definition (3.5) imply the desired time estimate (3.18), concluding the Proof of Theorem 3.2.

Thanks to the superposition principle, Theorems 2.1, 3.1, and 3.2 can be combined to infer Theorem 1.1 for the forced linear heat IBVP on the half-line (1.2). In particular, let $U_{0} \in H_{x}^{s}(\mathbb{R})$ and $F \in C\left([0, T] ; H_{x}^{s}(\mathbb{R})\right)$ be, respectively, whole-line extensions of the initial datum $u_{0} \in H_{x}^{s}(0, \infty)$ and the forcing $f \in C\left([0, T] ; H_{x}^{s}(0, \infty)\right)$ of problem (1.2) satisfying

$$
\left\|U_{0}\right\|_{H_{x}^{s}(\mathbb{R})} \leqslant c\left\|u_{0}\right\|_{H_{x}^{s}(0, \infty)}, \quad \sup _{t \in[0, T]}\|F(t)\|_{H_{x}^{s}(\mathbb{R})} \leqslant c \sup _{t \in[0, T]}\|f(t)\|_{H_{x}^{s}(0, \infty)} .
$$

Furthermore, let

$$
G_{0}(t)=g_{0}(t)-S\left[U_{0} ; 0\right](0, t)
$$

and note that, thanks to the compatibility condition $u_{0}(0)=g_{0}(0)$ of Theorem 1.1 , for $s>\frac{1}{2}$ we have $G_{0}(0)=0$. Then, by linearity, the solution of IBVP (1.2) can be expressed in the form

$$
S\left[u_{0}, g_{0} ; f\right]=\left.S\left[U_{0} ; 0\right]\right|_{x \in(0, \infty)}+\left.S[0 ; F]\right|_{x \in(0, \infty)}+S\left[0, G_{0} ; 0\right]-S\left[0,\left.S[0 ; F]\right|_{x=0} ; 0\right],
$$

where the first and second terms on the right-hand side are the solutions of problems (3.1) and (3.15), which have been estimated in Theorems 3.1 and 3.2 , while the third and fourth terms satisfy the IBVP 


$$
\begin{array}{ll}
u_{t}-u_{x x}=0, & x \in(0, \infty), t \in(0, T), \\
u(x, 0)=0, & x \in[0, \infty), \\
u(0, t)=G(t), & t \in[0, T],
\end{array}
$$

with $G=G_{0}$ and $G=\left.S[0 ; F]\right|_{x=0}$, respectively. Note that in both cases we have $G(0)=0$. Furthermore, the time estimates (3.4) and (3.18) together with inequalities (3.24) imply

$$
\begin{array}{rlrl}
\left\|G_{0}\right\|_{H_{t}^{\frac{2 s+1}{4}}(0, T)} & \leqslant c_{s}\left(\left\|u_{0}\right\|_{H_{x}^{s}(0, \infty)}+\left\|g_{0}\right\|_{H_{t}^{\frac{2 s+1}{4}}(0, T)}\right), & & 0 \leqslant s<\frac{3}{2}, \\
\left\|\left.S[0 ; F]\right|_{x=0}\right\|_{H_{t}^{\frac{2 s+1}{4}}(0, T)} \leqslant c_{s} \sqrt{T} \sup _{t \in[0, T]}\|f(t)\|_{H_{x}^{s}(0, \infty),} & \frac{1}{2}<s<\frac{3}{2},
\end{array}
$$

i.e., in both cases $G \in H_{t}^{(2 s+1) / 4}(0, T)$. Thus, along the lines of Ref. 11, $G$ can be extended outside $(0, T)$ via the compactly supported function

$$
g(t)= \begin{cases}E_{\theta}(t), & t \in(0,2), \\ 0, & t \in(0,2)^{c},\end{cases}
$$

where $E_{\theta}(t)=\theta(t) E(t)$ with $\theta \in C_{0}^{\infty}(\mathbb{R})$ a smooth cutoff function such that $0 \leqslant \theta(t) \leqslant 1$ for all $t \in \mathbb{R}, \theta(t)=1$ for $|t| \leqslant 1$, and $\theta(t)=0$ for $|t| \geqslant 2$, and $E \in H_{t}^{(2 s+1) / 4}(\mathbb{R})$ an extension of $G$ such that $\|E\|_{H_{t}^{(2 s+1) / 4}(\mathbb{R})} \leqslant c\|G\|_{H_{t}^{(2 s+1) / 4}(0, T)}$. Importantly, by construction, we have supp $(g) \subset(0,2)$ and

$$
\|g\|_{H_{t}^{\frac{2 s+1}{4}}(\mathbb{R})} \leqslant \mathcal{c}_{s}\|G\|_{H_{t}^{\frac{2 s+1}{4}}(0, T)}, \quad \frac{1}{2}<s<\frac{3}{2} .
$$

Thus, for the boundary datum $g$ defined by (3.29), the pure linear IBVP (2.1) restricted on $(0, \infty) \times(0, T)$ becomes precisely IBVP (3.27). Therefore, for $\frac{1}{2}<s<\frac{3}{2}$, Theorem 2.1 and inequalities (3.28) imply the space estimates

$$
\begin{aligned}
& \sup _{t \in[0, T]}\left\|S\left[0, G_{0} ; 0\right](t)\right\|_{H_{x}^{s}(0, \infty)} \leqslant c_{s}\left(\left\|u_{0}\right\|_{H_{x}^{s}(0, \infty)}+\left\|g_{0}\right\|_{H_{t}^{\frac{2 s+1}{4}}(0, T)}\right), \\
& \sup _{t \in[0, T]}\left\|S\left[0,\left.S[0 ; F]\right|_{x=0} ; 0\right](t)\right\|_{H_{x}^{s}(0, \infty)} \leqslant c_{s} \sqrt{T} \sup _{t \in[0, T]}\|f(t)\|_{H_{x}^{s}(0, \infty)},
\end{aligned}
$$

and the time estimates

$$
\begin{gathered}
\sup _{x \in[0, \infty)}\left\|S\left[0, G_{0} ; 0\right](x)\right\|_{H_{t}^{\frac{2 s+1}{4}}(0, T)} \leqslant c_{s}\left(\left\|u_{0}\right\|_{H_{x}^{s}(0, \infty)}+\left\|g_{0}\right\|_{H_{t}^{\frac{2 s+1}{4}}(0, T)}\right), \\
\sup _{x \in[0, \infty)}\left\|S\left[0,\left.S[0 ; F]\right|_{x=0} ; 0\right](x)\right\|_{H_{t}^{\frac{2 s+1}{4}}(0, T)} \leqslant c_{s} \sqrt{T} \sup _{t \in[0, T]}\|f(t)\|_{H_{x}^{s}(0, \infty)} .
\end{gathered}
$$

Estimates (3.31) and (3.32) combined with Theorems 3.1 and 3.2 and the superposition formula (3.26) yield Theorem 1.1.

Local well-posedness on the half-line. The linear estimates of Theorem 1.1 will now be combined with a contraction mapping argument in order to prove Theorem 1.2 on the well-posedness of the nonlinear reaction-diffusion IBVP on the half-line (1.1).

Existence and uniqueness of solution. We set $f=|u|^{p-1} u=u^{p}, \frac{p-1}{2} \in \mathbb{N}$, and use the unified transform formula (1.3) for the linear IBVP (1.2) to define the following iteration map for the nonlinear IBVP (1.1):

$$
u \mapsto \Phi u=\Phi_{u_{0}, g_{0}} u \doteq S\left[u_{0}, g_{0} ; u^{p}\right]
$$

We shall show that for lifespan $T^{*}$ given by (1.9), this map is a contraction in the space

$$
X=C\left(\left[0, T^{*}\right] ; H_{x}^{s}(0, \infty)\right) .
$$

The space estimate (1.6) together with the algebra property in $H_{x}^{s}(0, \infty)$ implies 


$$
\|\Phi u\|_{X} \leqslant c_{s}\left(\left\|\left(u_{0}, g_{0}\right)\right\|_{D}+\sqrt{T^{*}}\|u\|_{X}^{p}\right), \quad \frac{1}{2}<s<\frac{3}{2} .
$$

Let $B(0, \varrho)=\left\{u \in X:\|u\|_{X} \leqslant \varrho\right\}$ be a ball centered at 0 with radius $\varrho=2 c_{s}\left\|\left(u_{0}, g_{0}\right)\right\|_{D}$, where the data norm $\left\|\left(u_{0}, g_{0}\right)\right\|_{D}$ is defined in Theorem 1.2. Then, for $u \in B(0, \varrho)$, we have

$$
\|\Phi u\|_{X} \leqslant \frac{\varrho}{2}+c_{s} \sqrt{T^{*}} \varrho^{p} .
$$

Thus, the map $u \mapsto \Phi u$ takes $B(0, \varrho)$ into $B(0, \varrho)$, provided that

$$
\frac{\varrho}{2}+c_{s} \sqrt{T^{*}} \varrho^{p} \leqslant \varrho \Leftrightarrow T^{*} \leqslant \frac{1}{\left(2 c_{s}\right)^{2 p}\left\|\left(u_{0}, g_{0}\right)\right\|_{D}^{2(p-1)}} .
$$

Furthermore, using estimate (1.6) for $\Phi u_{1}-\Phi u_{2}=S\left[0,0 ; u_{1}^{p}-u_{2}^{p}\right]$, we obtain

$$
\left\|\Phi u_{1}-\Phi u_{2}\right\|_{X} \leqslant c_{s} \sqrt{T^{*}} \sup _{t \in\left[0, T^{*}\right]}\left\|\left(u_{1}^{p}-u_{2}^{p}\right)(t)\right\|_{H_{x}^{s}(0, \infty)}, \quad \frac{1}{2}<s<\frac{3}{2}
$$

Thus, employing the identity $u_{1}^{p}-u_{2}^{p}=\left(u_{1}^{p-1}+u_{1}^{p-2} u_{2}+\cdots+u_{1} u_{2}^{p-2}+u_{2}^{p-1}\right)\left(u_{1}-u_{2}\right)$ and the algebra property in $H_{x}^{s}(0$, $\infty)$, we deduce

$$
\left\|\Phi u_{1}-\Phi u_{2}\right\|_{X} \leqslant p c_{s} \sqrt{T^{\star}} e^{p-1}\left\|u_{1}-u_{2}\right\|_{X}, \quad \frac{1}{2}<s<\frac{3}{2} .
$$

Hence, the map $u \mapsto \Phi u$ is a contraction in $B(0, \varrho)$ with

$$
\left\|\Phi u_{1}-\Phi u_{2}\right\|_{X} \leqslant \frac{1}{2}\left\|u_{1}-u_{2}\right\|_{X}, \quad \forall u_{1}, u_{2} \in B(0, \varrho)
$$

provided that

$$
p c_{s} \sqrt{T^{*}} Q^{p-1} \leqslant \frac{1}{2} \Leftrightarrow T^{*} \leqslant \frac{1}{p^{2}\left(2 c_{s}\right)^{2 p}\left\|\left(u_{0}, g_{0}\right)\right\|_{D}^{2(p-1)}} .
$$

Since $p>1$, condition (3.34) is weaker than condition (3.36) and they are both satisfied for $T^{*}$ given by (1.9). Therefore, by the contraction mapping theorem, the map $u \mapsto \Phi u$ has a unique fixed point in $B(0, \varrho)$. Equivalently, the integral equation $u=\Phi u$ for the reaction-diffusion IBVP (1.1) has a unique solution $u \in B(0, \varrho) \subset X$.

Finally, we note that thanks to the time estimate (1.7) the contraction mapping argument can also be carried out in the finer space $C\left(\left[0, T^{*}\right] ; H_{x}^{s}(0, \infty)\right) \cap C\left([0, \infty) ; H_{t}^{(2 s+1) / 4}\left(0, T^{*}\right)\right)$ instead of just the space $X$, thus giving rise to estimate (1.8). The proof of existence and uniqueness is complete.

Continuity of the data-to-solution map. We conclude the Proof of Theorem 1.2 by showing that the data-to-solution map $H_{x}^{s}(0, \infty)$ $\times H_{t}^{(2 s+1) / 4}(0, T) \ni\left(u_{0}, g_{0}\right) \mapsto u \in X$ is continuous. Let $\left(u_{0_{1}}, g_{0_{1}}\right)$ and $\left(u_{0_{2}}, g_{0_{2}}\right)$ be two pairs of data lying inside a ball $B_{\delta} \subset D$ of radius $\delta>0$ centered at a distance $a$ from the origin. Denote by $u_{1}=\Phi_{u_{0_{1}}, g_{0_{1}}} u_{1}$ and $u_{2}=\Phi_{u_{0_{2}}, g_{02}} u_{2}$ the corresponding solutions to IBVP (1.1), which are obtained as fixed points in the spaces $X_{u_{1}}$ and $X_{u_{2}}$ defined by (3.33) with $T^{*}$ replaced by the relevant lifespans $T_{u_{1}}$ and $T_{u_{2}}$ according to (1.9). Since $\max \left\{\left\|\left(u_{0_{1}}, g_{0_{1}}\right)\right\|_{D},\left\|\left(u_{0_{2}}, g_{0_{2}}\right)\right\|_{D}\right\} \leqslant a+\delta$ and $p>1$, it follows that

$$
\min \left\{T_{u_{1}}, T_{u_{2}}\right\} \geqslant \min \left\{T, \frac{1}{p^{2}\left(2 c_{s}\right)^{2 p}(a+\delta)^{2(p-1)}}\right\} \doteq T_{c} .
$$

Hence, both $u_{1}$ and $u_{2} \leqslant t \leqslant T_{c}$. The common lifespan $T_{c}$ gives rise to the space $X_{c}=C\left(\left[0, T_{c}\right] ; H_{x}^{s}(0, \infty)\right)$. We shall now determine the radius $\varrho_{c}$ of a ball $B\left(0, \varrho_{c}\right) \subset X_{c}$ such that $u_{1}, u_{2} \in B\left(0, \varrho_{c}\right)$ and

$$
\left\|u_{1}-u_{2}\right\|_{X_{c}} \leqslant 2 c_{s}\left\|\left(u_{0_{1}}-u_{0_{2}}, g_{0_{1}}-g_{0_{2}}\right)\right\|_{D}
$$

Observe that $X_{u_{1}}, X_{u_{2}} \subseteq X_{c}$ and hence $u_{1}, u_{2}$ are fixed points of $\Phi$ in $X_{c}$. Thus, 


$$
\left\|u_{1}-u_{2}\right\|_{X_{c}}=\left\|\Phi u_{1}-\Phi u_{2}\right\|_{X_{c}}=\left\|S\left[u_{0_{1}}-u_{0_{2}}, g_{0_{1}}-g_{0_{2}} ; u_{1}^{p}-u_{2}^{p}\right]\right\|_{X_{c}}
$$

and estimate (1.6) together with the algebra property in $H_{x}^{s}(0, \infty)$ implies

$$
\left\|u_{1}-u_{2}\right\|_{X_{c}} \leqslant c_{s}\left\|\left(u_{0_{1}}-u_{0_{2}}, g_{0_{1}}-g_{0_{2}}\right)\right\|_{D}+p c_{s} \sqrt{T_{c}} \varrho_{c}^{p-1}\left\|u_{1}-u_{2}\right\|_{X_{c}} .
$$

Choosing $\varrho_{c}=2 c_{s}(a+\delta)$ turns the above into the desired inequality (3.37), implying local Lipschitz continuity of the data-to-solution map. The Proof of Theorem 1.2 is complete.

\section{THE CASE OF A FINITE INTERVAL: PROOF OF THEOREM 1.3}

As in the case of the half-line, the analysis of the reaction-diffusion IBVP on a finite interval (1.10) depends crucially on the study of the associated forced linear IBVP, namely,

$$
\begin{array}{ll}
u_{t}-u_{x x}=f(x, t), & x \in(0, \ell), t \in(0, T), \\
u(x, 0)=u_{0}(x), & x \in[0, \ell], \\
u(0, t)=g_{0}(t), u(\ell, t)=h_{0}(t), & t \in[0, T] .
\end{array}
$$

Actually, the analysis of problem (4.1) can be simplified significantly by exploiting the results of Theorem 1.1 for the linear half-line IBVP. More specifically, we let $U_{0} \in H_{x}^{s}(\mathbb{R})$ and $F \in C\left([0, T] ; H_{x}^{s}(\mathbb{R})\right)$ be whole-line extensions of the initial datum $u_{0} \in H_{x}^{s}(0, \ell)$ and the forcing $f \in C\left([0, T] ; H_{x}^{s}(0, \ell)\right)$ such that $\left\|U_{0}\right\|_{H_{x}^{s}(\mathbb{R})} \leqslant c\left\|u_{0}\right\|_{H_{x}^{s}(0, \ell)}$ and $\|F\|_{C\left([0, T] ; H_{x}^{s}(\mathbb{R})\right)} \leqslant c\|f\|_{C\left([0, T] ; H_{x}^{s}(0, \ell)\right)}$. Then, the solution of problem (4.1) can be expressed as

$$
S\left[u_{0}, g_{0}, h_{0} ; f\right]=\left.S\left[\left.U_{0}\right|_{x \in[0, \infty)}, g_{0} ;\left.F\right|_{x \in[0, \infty)}\right]\right|_{x \in[0, \ell]}+S\left[0,0, w_{0} ; 0\right],
$$

where $S\left[\left.U_{0}\right|_{x \in[0, \infty)}, g_{0} ;\left.F\right|_{x \in[0, \infty)}\right]$ is the solution of the linear half-line IBVP (1.2) restricted on the interval $[0, \ell]$ and $S\left[0,0, w_{0} ; 0\right]$ satisfies the reduced finite interval problem,

$$
\begin{array}{ll}
u_{t}-u_{x x}=0, & x \in(0, \ell), t \in(0, T), \\
u(x, 0)=0, & x \in[0, \ell], \\
u(0, t)=0, u(\ell, t)=w_{0}(t), & t \in[0, T],
\end{array}
$$

with

$$
w_{0}(t) \doteq h_{0}(t)-S\left[\left.U_{0}\right|_{x \in[0, \infty)}, g_{0} ;\left.F\right|_{x \in[0, \infty)}\right](\ell, t) .
$$

Since the half-line solution $S\left[\left.U_{0}\right|_{x \in[0, \infty)}, g_{0} ;\left.F\right|_{x \in[0, \infty)}\right]$ have already been treated in Theorem 1.1, relation (4.2) reduces the analysis of the finite interval IBVP (4.1) to that of problem (4.3).

Note that for $\frac{1}{2}<s<\frac{3}{2}$ the half-line estimate (1.7) combined with the inequalities for $U_{0}$ and $F$ stated above implies that $w_{0} \in H_{t}^{(2 s+1) / 4}(0, T)$ with

$$
\left\|w_{0}\right\|_{H_{t}^{\frac{2 s+1}{4}}(0, T)} \leqslant c_{s}\left(\left\|u_{0}\right\|_{H_{x}^{s}(0, \ell)}+\left\|g_{0}\right\|_{H_{t}^{\frac{2 s+1}{4}}(0, T)}+\left\|h_{0}\right\|_{H_{t}^{\frac{2 s+1}{4}}(0, T)}+\sqrt{T} \sup _{t \in[0, T]}\|f(t)\|_{H_{x}^{s}(0, \ell)}\right)
$$

Furthermore, observe that $w_{0}(0)=h_{0}(0)-u_{0}(\ell)=0$ thanks to the second compatibility condition stated in Theorem 1.3. Thus, in analogy to the half-line, IBVP (4.3) can be embedded in the pure linear IBVP,

$$
\begin{array}{ll}
v_{t}-v_{x x}=0, & x \in(0, \ell), t \in(0,2), \\
v(x, 0)=0, & x \in[0, \ell], \\
v(0, t)=0, v(\ell, t)=h(t) \in H_{t}^{\frac{2 s+1}{4}}(\mathbb{R}), & t \in[0,2],
\end{array}
$$

where $h$ is an extension of $w_{0}$ constructed analogously to (3.29) so that $\operatorname{supp}(h) \subset(0,2)$ and

$$
\|h\|_{H_{t}^{\frac{2 s+1}{4}}(\mathbb{R})} \leqslant c_{s}\left\|w_{0}\right\|_{H_{t}^{\frac{2 s+1}{4}}(0, T)}, \quad \frac{1}{2}<s<\frac{3}{2} .
$$


Hence, problem (4.3) can be estimated via problem (4.6), whose unified transform solution formula is ${ }^{10}$

$$
\begin{aligned}
v(x, t)= & S[0,0, h ; 0](x, t) \\
= & \frac{1}{\pi} \int_{k=0}^{\infty} \frac{e^{i a^{3} k x+i k^{2} t}}{e^{i a^{3} k \ell}-e^{-i a^{3} k \ell}} \widehat{h}\left(k^{2}\right) d k+\frac{1}{\pi} \int_{k=0}^{\infty} \frac{e^{i a k x-i k^{2} t}}{e^{i a k \ell}-e^{-i a k \ell}} k \widehat{h}\left(-k^{2}\right) d k \\
& +\frac{1}{\pi} \int_{k=0}^{\infty} \frac{e^{-i a^{3} k x+i k^{2} t}}{e^{-i a^{3} k \ell}-e^{i a^{3} k \ell}} \widehat{h}\left(k^{2}\right) d k+\frac{1}{\pi} \int_{k=0}^{\infty} \frac{e^{-i a k x-i k^{2} t}}{e^{-i a k \ell}-e^{i a k \ell}} k \widehat{h}\left(-k^{2}\right) d k,
\end{aligned}
$$

where $a=e^{i \frac{\pi}{4}}, \widehat{h}$ is the whole-line Fourier transform of $h$ (this is possible thanks to the compact support of $h$ ), and the contours $\partial D^{ \pm}$are depicted in Fig. 1. Using formula (4.8), we shall prove the following result for the pure linear IBVP (4.6):

Theorem 4.1 (Pure linear IBVP on a finite interval). The solution $v=S[0,0, h ; 0]$ of the pure linear IBVP (4.6) given by the unified transform formula (4.8) satisfies the space and time estimates,

$$
\begin{array}{cl}
\sup _{t \in[0,2]}\|v(t)\|_{H_{x}^{s}(0, \ell)} \leqslant c_{s}\|h\|_{H_{t}^{\frac{2 s+1}{4}}(\mathbb{R})}, & s \geqslant 0, \\
\sup _{x \in[0, \ell]}\|v(x)\|_{H_{t}^{\frac{2 s+1}{4}}(0,2)} \leqslant c_{s}\|h\|_{H_{t}^{\frac{2 s+1}{4}}(\mathbb{R})}, & s \in \mathbb{R} .
\end{array}
$$

Proof of Theorem 4.1. The main ideas behind the proof are the same with those that form the backbone of the proof of the halfline Theorem 2.1. Now, however, extra care is needed when handling the differences of exponentials appearing in the denominators of formula (4.8).

We begin with the space estimate (4.9). Grouping together the first with the third and the second with the fourth term of (4.8), we decompose $v=v_{1}+v_{2}$ where

$$
\begin{array}{ll}
v_{1}(x, t)=\int_{k=0}^{\infty} \frac{e^{i \gamma_{1} k x}-e^{-i \gamma_{1} k x}}{e^{i \gamma_{1} k \ell}-e^{-i \gamma_{1} k \ell}} G_{1}(k, t) d k, & \gamma_{1}=a^{3}, G_{1}(k, t)=\frac{1}{\pi} e^{i k^{2} t} k \widehat{h}\left(k^{2}\right), \\
v_{2}(x, t)=\int_{k=0}^{\infty} \frac{e^{i \gamma_{2} k x}-e^{-i \gamma_{2} k x}}{e^{i \gamma_{2} k \ell}-e^{-i \gamma_{2} k \ell}} G_{2}(k, t) d k, & \gamma_{2}=a, G_{2}(k, t)=\frac{1}{\pi} e^{-i k^{2} t} k \widehat{h}\left(-k^{2}\right) .
\end{array}
$$

The estimation of $v_{1}$ and $v_{2}$ is analogous so we only provide the details for $v_{1}$. We conveniently use the physical space definition of the $H_{x}^{s}(0, \ell)$ -norm,

$$
\left\|v_{1}(t)\right\|_{H_{x}^{s}(0, \ell)}=\sum_{j=0}^{\lfloor s\rfloor}\left\|\partial_{x}^{j} v_{1}(t)\right\|_{L_{x}^{2}(0, \ell)}+\left\|\partial_{x}^{\lfloor s\rfloor} v_{1}(t)\right\|_{\beta}, \quad s=\lfloor s\rfloor+\beta \geqslant 0,0 \leqslant \beta<1,
$$

where $[\cdot]$ denotes the floor function and the fractional norm $\|\cdot\|_{\beta}$ is defined by

$$
\left\|v_{1}(t)\right\|_{\beta}^{2}=\int_{x=0}^{\ell} \int_{z=0}^{\ell-x} \frac{\left|v_{1}(x+z, t)-v_{1}(x, t)\right|^{2}}{z^{1+2 \beta}} d z d x, \quad 0<\beta<1 .
$$

(i) The case $\lfloor s\rfloor=0$. Since $s=\beta \in[0,1)$, we only need to estimate $\left\|v_{1}(t)\right\|_{\beta}$ and $\left\|v_{1}(t)\right\|_{L_{x}^{2}(0, \ell)}$. For the fractional norm $\left\|v_{1}(t)\right\|_{\beta}$, we write

$$
v_{1}(x, t)=J_{1}(x, t)+J_{2}(x, t),
$$

with

For $J_{1}$, we have

$$
J_{1}(x, t)=\int_{k=0}^{1} \frac{e^{i \gamma_{1} k x}-e^{-i \gamma_{1} k x}}{e^{i \gamma_{1} k \ell}-e^{-i \gamma_{1} k \ell}} G_{1}(k, t) d k, \quad J_{2}(x, t)=\int_{k=1}^{\infty} \frac{e^{i \gamma_{1} k x}-e^{-i \gamma_{1} k x}}{e^{i \gamma_{1} k \ell}-e^{-i \gamma_{1} k \ell}} G_{1}(k, t) d k
$$

$$
\begin{aligned}
\left\|J_{1}(t)\right\|_{\beta} & =\left(\int_{z=0}^{\ell} \int_{x=0}^{\ell-z} \frac{1}{z^{1+2 \beta}}\left|\int_{k=0}^{1} \frac{\left(e^{i \gamma_{1} k z}-1\right)\left[e^{i \gamma_{1} k(x+\ell)}+e^{-i \gamma_{1} k(x+z-\ell)}\right]}{e^{2 i \gamma_{1} k \ell}-1} G_{1}(k, t) d k\right|^{2} d x d z\right)^{\frac{1}{2}} \\
& \leqslant \int_{k=0}^{1}\left(\int_{z=0}^{\ell} \int_{x=0}^{\ell-z} \frac{1}{z^{1+2 \beta}} \frac{\left|1-e^{i \gamma_{1} k z}\right|^{2}}{\left|1-e^{2 i \gamma_{1} k \ell}\right|^{2}}\left|G_{1}(k, t)\right|^{2} d x d z\right)^{\frac{1}{2}} d k
\end{aligned}
$$


after noting that $x+\ell \geqslant 0$ and $x+z-\ell=x-(\ell-z) \leqslant 0$ and applying Minkowski's integral inequality in $k$. Hence, using Lemma 2.1 , integrating in $x$ and employing (2.9), we obtain

$$
\left\|J_{1}(t)\right\|_{\beta} \lesssim \sqrt{\ell} \int_{k=0}^{1} \frac{\left|G_{1}(k, t)\right|}{1-e^{-\sqrt{2} k \ell}}\left(\int_{z=0}^{\ell} \frac{\left(1-e^{-\frac{\sqrt{2}}{2} k z}\right)^{2}}{z^{1+2 \beta}} d z\right)^{\frac{1}{2}} d k \lesssim \int_{k=0}^{1} \frac{k^{\frac{1}{2}+\beta}}{1-e^{-\sqrt{2} k \ell}} k^{\frac{1}{2}}\left|\widehat{h}\left(k^{2}\right)\right| d k
$$

Then, applying the Cauchy-Schwarz inequality and noting that $\int_{k=0}^{1} \frac{k^{1+2 \beta}}{\left(1-e^{-\sqrt{2} k \ell}\right)^{2}} d k \lesssim 1$ since the singularity at $k=0$ is removable and the domain of integration is compact, we deduce

$$
\left\|J_{1}(t)\right\|_{\beta} \lesssim\|h\|_{L_{t}^{2}(\mathbb{R})} \leqslant\|h\|_{H_{t}^{\frac{2 \beta+1}{4}}(\mathbb{R})}, \quad 0<\beta<1
$$

Regarding $J_{2}$, writing $J_{2}=J_{21}+J_{22}$ with

$$
\begin{array}{ll}
J_{21}(x, t)=\int_{k=1}^{\infty} e^{i a^{3} k x} K_{1}(k, t) d k, & K_{1}(k, t)=\frac{G_{1}(k, t)}{e^{i a^{3} k \ell}-e^{-i a^{3} k \ell},} \\
J_{22}(x, t)=\int_{k=1}^{\infty} e^{-i a^{3} k(x-\ell)} K_{2}(k, t) d k, & K_{2}(k, t)=\frac{G_{1}(k, t)}{1-e^{2 i a^{3} k \ell}}
\end{array}
$$

and employing once again Lemma 2.1, we infer

$$
\left\|J_{21}(t)\right\|_{\beta}^{2} \lesssim \int_{z=0}^{\ell} \frac{1}{z^{1+2 \beta}}\left\|\int_{k=1}^{\infty} e^{-\frac{\sqrt{2}}{2} k x}\left(1-e^{-\frac{\sqrt{2}}{2} k z}\right)\left|K_{1}(k, t)\right| d k\right\|_{L_{x}^{2}(0, \infty)}^{2} d z
$$

Hence, combining the Laplace transform Lemma 2.2 with (2.9), we find

$$
\left\|J_{21}(t)\right\|_{\beta}^{2} \lesssim \int_{k=1}^{\infty} \frac{k^{2}\left|\widehat{h}\left(k^{2}\right)\right|^{2} k^{2 \beta}}{\left|e^{i a^{3} k \ell}-e^{-i a^{3} k \ell}\right|^{2}} d k \lesssim \int_{k=1}^{\infty} \frac{\left.k^{2} \sqrt{h}\left(k^{2}\right)\right|^{2} k^{2 \beta}}{\left(e^{\frac{\sqrt{2}}{2} k \ell}-e^{-\frac{\sqrt{2}}{2} k \ell}\right)^{2}} d k
$$

with the last inequality due to the fact that $\left|e^{i a^{3} k \ell}-e^{-i a^{3} k \ell}\right| \geqslant e^{\frac{\sqrt{2}}{2} k \ell}-e^{-\frac{\sqrt{2}}{2} k \ell}$ for $k \geqslant 0$.

Next, let $\psi(k)=e^{\frac{\sqrt{2}}{2} k \ell}-e^{-\frac{\sqrt{2}}{2} k \ell}$ and note that $\psi(k)>0$ for $k \geqslant 1$ [importantly, $\psi(k)=0$ only for $k=0$, i.e., the integrand of (4.14) is non-singular]. Moreover, observe that $\psi$ is infinitely differentiable and, in particular, $\psi$ is increasing on $[1, \infty)$. Hence, $0<e^{\frac{\sqrt{2}}{2} \ell}-e^{-\frac{\sqrt{2}}{2} \ell}$ $\leqslant e^{\frac{\sqrt{2}}{2} k \ell}-e^{-\frac{\sqrt{2}}{2} k \ell}$ for $k \geqslant 1$. Thus, back to (4.14), we have

$$
\left\|J_{21}(t)\right\|_{\beta}^{2} \lesssim \int_{k=1}^{\infty} \frac{k^{2}\left|\widehat{h}\left(k^{2}\right)\right|^{2} k^{2 \beta}}{\left(e^{\frac{\sqrt{2}}{2} \ell}-e^{-\frac{\sqrt{2}}{2} \ell}\right)^{2}} d k \simeq \int_{k=1}^{\infty} k^{1+2 \beta}\left|\widehat{h}\left(k^{2}\right)\right|^{2} k d k \lesssim\|h\|_{H_{t}^{2}}^{2}(\mathbb{R})
$$

Concerning $J_{22}$, we note that $\left\|J_{22}(t)\right\|_{\beta}=\left\|\widetilde{J}_{22}(t)\right\|_{\beta}$, where $\widetilde{J}_{22}(x, t) \doteq J_{22}(\ell-x, t)$. Therefore,

$$
\left\|J_{22}(t)\right\|_{\beta}^{2}=\left\|\widetilde{J}_{22}(t)\right\|_{\beta}^{2} \lesssim \int_{x=0}^{\ell} \int_{z=0}^{\ell-x} \frac{1}{z^{1+2 \beta}}\left(\int_{k=1}^{\infty} e^{-\frac{\sqrt{2}}{2} k x}\left|e^{i a^{3} k z}-1\right|\left|K_{2}(k, t)\right| d k\right)^{2} d z d x
$$

and employing once again Lemma 2.1, we infer

$$
\left\|J_{22}(t)\right\|_{\beta}^{2} \lesssim \int_{z=0}^{\ell} \frac{1}{z^{1+2 \beta}}\left\|\int_{k=1}^{\infty} e^{-\frac{\sqrt{2}}{2} k x}\left(1-e^{-\frac{\sqrt{2}}{2} k z}\right)\left|K_{2}(k, t)\right| d k\right\|_{L_{x}^{2}(0, \infty)}^{2} d z
$$

The Laplace transform Lemma 2.2, estimate (2.9), and the fact that $\left|1-e^{2 i a^{3} k \ell}\right| \geqslant 1-e^{-\sqrt{2} k \ell}$ for all $k \geqslant 0$ imply 


$$
\left\|J_{22}(t)\right\|_{\beta}^{2} \lesssim \int_{z=0}^{\frac{\sqrt{2}}{2} \ell} \frac{1}{z^{1+2 \beta}}\left\|Q_{z, t}\right\|_{L_{k}^{2}(0, \infty)}^{2} d z \lesssim \int_{k=1}^{\infty} \frac{k^{2}\left|\widehat{h}\left(k^{2}\right)\right|^{2}}{\left(1-e^{-\sqrt{2} k \ell}\right)^{2}} k^{2 \beta} d k .
$$

Similarly to the argument used for $J_{21}$, for $k \geqslant 1$, we have $0<1-e^{-\sqrt{2} \ell} \leqslant 1-e^{-\sqrt{2} k \ell}$. Hence,

$$
\left\|J_{22}(t)\right\|_{\beta}^{2} \lesssim \int_{k=1}^{\infty} \frac{k^{2}\left|\widehat{h}\left(k^{2}\right)\right|^{2}}{\left(1-e^{-\sqrt{2} \ell}\right)^{2}} k^{2 \beta} d k \leqslant \int_{k=1}^{\infty} k^{1+2 \beta}\left|\widehat{h}\left(k^{2}\right)\right|^{2} k d k \lesssim\|h\|_{H_{t}^{2 \beta+1}}^{2}(\mathbb{R})
$$

Overall, combining estimates (4.13), (4.15), and (4.16) with the writing (4.12), we find

$$
\left\|v_{1}(t)\right\|_{\beta} \lesssim\|h\|_{H_{t}^{\frac{2 s+1}{4}}(\mathbb{R})}, \quad s=\beta \in(0,1) .
$$

The norm $\left\|v_{1}(t)\right\|_{L_{x}^{2}(0, \ell)}$ will also be estimated using the splitting (4.12). In particular, for $J_{1}$, we employ Lemma 2.1 to infer

$$
\left\|J_{1}(t)\right\|_{L_{x}^{2}(0, \ell)}^{2} \lesssim \int_{x=0}^{\ell}\left(\int_{k=0}^{1} \frac{e^{\frac{\sqrt{2}}{2} k x}-e^{-\frac{\sqrt{2}}{2} k x}}{\mid e^{i a^{3} k \ell}-e^{-i a^{3} k \ell \mid}} k\left|\widehat{h}\left(k^{2}\right)\right| d k\right)^{2} d x .
$$

The ratio of exponentials involved in the $k$-integral is bounded by 1 for all $k \geqslant 0$. Thus, applying also the Cauchy-Schwarz inequality in $k$, we obtain

$$
\left\|J_{1}(t)\right\|_{L_{x}^{2}(0, \ell)}^{2} \lesssim \int_{x=0}^{\ell}\left(\int_{\tau=0}^{1}|\widehat{h}(\tau)|^{2} d \tau\right) d x \lesssim\|h\|_{H_{t}^{2}}^{2}(\mathbb{R}), \quad s \geqslant-\frac{1}{2} .
$$

Concerning $J_{2}$, similarly to (4.18), we have

$$
\left\|J_{2}(t)\right\|_{L_{x}^{2}(0, \ell)}^{2} \lesssim \int_{x=0}^{\ell}\left(\frac{1}{\pi} \int_{k=1}^{\infty} \frac{e^{\frac{\sqrt{2}}{2} k x}-e^{-\frac{\sqrt{2}}{2} k x}}{\left|e^{i a^{3} k \ell}-e^{-i a^{3} k \ell}\right|} k\left|\widehat{h}\left(k^{2}\right)\right| d k\right)^{2} d x .
$$

Note that $\left|e^{i a^{3} k \ell}-e^{-i a^{3} k \ell}\right| \geqslant e^{\frac{\sqrt{2}}{2} k \ell}-e^{-\frac{\sqrt{2}}{2} k \ell}$ and, furthermore,

$$
\frac{1}{e^{\frac{\sqrt{2}}{2} k \ell}-e^{-\frac{\sqrt{2}}{2} k \ell}} \leqslant \frac{e^{-\frac{\sqrt{2}}{2} k \ell}}{1-e^{-\sqrt{2} \ell}}, \quad k \geqslant 1 .
$$

Using this bound and the fact that $e^{-\frac{\sqrt{2}}{2} k \ell}<1$ for all $k \geqslant 1$, we find

$$
\begin{aligned}
\left\|J_{2}(t)\right\|_{L_{x}^{2}(0, \ell)}^{2} & \lesssim \int_{x=0}^{\ell}\left(\int_{k=1}^{\infty}\left(e^{\frac{\sqrt{2}}{2} k x}-e^{-\frac{\sqrt{2}}{2} k x}\right) e^{-\frac{\sqrt{2}}{2} k \ell} k \sqrt{h}\left(k^{2}\right) \mid d k\right)^{2} d x \\
& \lesssim \int_{x=0}^{\frac{\sqrt{2}}{2} \ell}\left(\int_{k=1}^{\infty} e^{-k x} k\left|\widehat{h}\left(k^{2}\right)\right| d k\right)^{2} d x \leqslant \int_{x=0}^{\infty}\left(\int_{k=1}^{\infty} e^{-k x} k\left|\widehat{h}\left(k^{2}\right)\right| d k\right)^{2} d x .
\end{aligned}
$$

Therefore, by the Laplace transform Lemma 2.2, we get

$$
\left.\left\|J_{2}(t)\right\|_{L_{x}^{2}(0, \ell) \lesssim}^{2} \int_{k=1}^{\infty} k^{2} \widehat{h}\left(k^{2}\right)\right|^{2} d k=\int_{\tau=1}^{\infty} \tau^{\frac{1}{2}}|\widehat{h}(\tau)|^{2} d \tau \leqslant\|h\|_{H_{t}^{\frac{2 s+1}{4}(\mathbb{R})}}^{2}, \quad s \geqslant 0 .
$$

Combining (4.19) and (4.21), we obtain

$$
\left\|v_{1}(t)\right\|_{L_{x}^{2}(0, \ell)} \lesssim\|h\|_{H_{t}^{\frac{2 s+1}{4}}(\mathbb{R})}, \quad s \geqslant 0 .
$$

(ii) The case $\lfloor s\rfloor>0$. Now $s=\lfloor s\rfloor+\beta$ with $\lfloor s\rfloor \in \mathbb{N} \backslash\{0\}$ and $\beta \in[0,1$ ). Thus, according to definition (4.11), we need to estimate the fractional norm $\left\|\partial_{x}^{[s\rfloor} v_{1}(t)\right\|_{\beta}$ and also the $L^{2}$-norm $\left\|\partial_{x}^{j} v_{1}(t)\right\|_{L_{x}^{2}(0, \ell)}$ for all integers $0 \leqslant j \leqslant\lfloor s\rfloor$. Both of those norms can be handled in exactly 
the same way as the norms $\left\|v_{1}(t)\right\|_{\beta}$ and $\left\|v_{1}(t)\right\|_{L_{x}^{2}(0, \ell)}$ that were estimated in case (i) above, eventually yielding the space estimate (4.9) for $v_{1}$ for all $s \geqslant 0$. As noted earlier, the estimation of $v_{2}$ is similar. Concerning the time estimate (4.10), the change of variable $k=\sqrt{\tau}$ in formula (4.8) reveals the temporal Fourier transform of $v$, which is then used to infer

$$
\begin{aligned}
& \|v(x)\|_{H_{t}^{\frac{2 s+1}{4}}(\mathbb{R})}^{2} \lesssim \int_{\tau=0}^{\infty}\left(1+\tau^{2}\right)^{\frac{2 s+1}{4}}\left|\frac{e^{i a^{3} \sqrt{\tau} x}-e^{-i a^{3} \sqrt{\tau} x}}{e^{i a^{3} \sqrt{\tau} \ell}-e^{-i a^{3} \sqrt{\tau} \ell}} \widehat{h}(\tau)\right|^{2} d \tau \\
& +\int_{\tau=-\infty}^{0}\left(1+\tau^{2}\right)^{\frac{2 s+1}{4}}\left|\frac{e^{i a \sqrt{-\tau} x}-e^{-i a \sqrt{-\tau} x}}{e^{i a \sqrt{-\tau} \ell}-e^{-i a \sqrt{-\tau} \ell}} \widehat{h}(\tau)\right|^{2} d \tau .
\end{aligned}
$$

Lemma 2.1 and the fact that $k \geqslant 0$ imply that the ratios of exponentials in the above integrals are bounded by 1 , and hence we arrive at the desired estimate (4.10).

Overall, Theorems 1.1 and 4.1 combined with inequalities (4.5) and (4.7) and the superposition (4.2) yield the following result for the forced linear IBVP on a finite interval (4.1):

Theorem 4.2 (Linear estimates on a finite interval). Suppose $\frac{1}{2}<s<\frac{3}{2}, u_{0} \in H_{x}^{s}(0, \ell), g_{0} \in H_{t}^{(2 s+1) / 4}(0, T)$, and $h_{0} \in H_{t}^{(2 s+1) / 4}(0, T)$ with $u_{0}(0)=g_{0}(0)$ and $u_{0}(\ell)=h_{0}(0)$. Then, the solution $u=S\left[u_{0}, g_{0}, h_{0} ; f\right]$ to the forced linear heat IBVP (4.1) satisfies

$$
\begin{aligned}
& \sup _{t \in[0, T]}\|u(t)\|_{H_{x}^{s}(0, \ell)}+\sup _{x \in[0, \ell]}\|u(x)\|_{H_{t}^{\frac{2 s+1}{4}}(0, T)} \\
\leqslant & c_{s}\left(\left\|u_{0}\right\|_{H_{x}^{s}(0, \ell)}+\left\|g_{0}\right\|_{H_{t}^{\frac{2 s+1}{4}}(0, T)}+\left\|h_{0}\right\|_{H_{t}^{\frac{2 s+1}{4}}(0, T)}+\sqrt{T} \sup _{t \in[0, T]}\|f(t)\|_{H_{x}^{s}(0, \ell)}\right) .
\end{aligned}
$$

Local well-posedness on a finite interval. The linear estimates of Theorem 4.2 allow us to prove Theorem 1.3 for the reaction-diffusion equation on a finite interval (1.10) along the lines of the contraction mapping argument of Sec. III. Indeed, the only modification required in the half-line proof of Sec. III is the replacement of the solution space $X$ and of the data norm $\|\cdot\|_{D}$ by their finite interval counterparts that are stated in Theorem 1.3.

\section{ROUGH DATA AND CONCLUDING REMARKS}

In Secs. II-IV, it was shown that the unified transform approach for showing well-posedness of nonlinear IBVPs is universal, in the sense that it can be employed not only for dispersive equations such as NLS and KdV but also for diffusive ones such as the reaction-diffusion equation (1.1a). Indeed, Theorem 1.2 provides the direct analog of the corresponding results for NLS ${ }^{11}$ and KdV, ${ }^{12}$ establishing local wellposedness in Sobolev spaces with $s>\frac{1}{2}$. Moreover, Theorem 1.3 advances the unified transform approach from the half-line to a finite interval.

In fact, the unified transform approach can also be employed for "rough" Sobolev data corresponding to $s<\frac{1}{2}$. In this case, the solution space $C\left([0, T] ; H_{x}^{s}(0, \infty)\right)$ of Theorem 1.2 must be refined by intersecting it with the space

$$
C^{\alpha}\left([0, T] ; L_{x}^{p}(0, \infty)\right)=\left\{u \in C\left([0, T] ; L_{x}^{p}(0, \infty)\right): \sup _{t \in[0, T]} t^{\alpha}\|u(t)\|_{L_{x}^{p}(0, \infty)}<\infty\right\},
$$

where $\alpha=\frac{1}{p}\left(\frac{1}{2}-b\right)$ with $\frac{2 s+1}{4}<b<\frac{1}{2}$. The presence of this new space helps us bypass the lack of the algebra property in $H_{x}^{s}(0, \infty)$ when $s<\frac{1}{2}$. In addition, in doing so, it removes the condition $\frac{p-1}{2} \in \mathbb{N}$, allowing for nonlinearities of any integer order $p \geqslant 2$. Our result reads as follows:

Theorem 5.1 (Well-posedness on the half-line for rough data). Suppose $\frac{1}{2}-\frac{1}{p}<s<\frac{1}{2}$ and $p=2,3,4, \ldots$ Then, for $u_{0} \in H_{x}^{s}(0, \infty)$ and $g_{0} \in H_{t}^{(2 s+1) / 4}(0, T)$, the reaction-diffusion IBVP (1.1) has a unique solution $u \in C\left(\left[0, T^{*}\right] ; H_{x}^{s}(0, \infty)\right) \cap C^{\alpha}\left(\left[0, T^{*}\right] ; L_{x}^{p}(0, \infty)\right)$ which satisfies

$$
\sup _{t \in\left[0, T^{*}\right]}\|u(t)\|_{H_{x}^{s}(0, \infty)}+\sup _{x \in[0, \infty)}\|u(x)\|_{H_{t}^{\frac{2 s+1}{4}}\left(0, T^{*}\right)}+\sup _{t \in\left[0, T^{*}\right]} t^{\alpha}\|u(t)\|_{L_{x}^{p}(0, \infty)} \leqslant 2 c_{s}\left\|\left(u_{0}, g_{0}\right)\right\|_{D}
$$

where $T^{*}=\min \left\{T, \frac{1}{\left(2^{p+2} p\right)^{\frac{1}{\alpha}}\left(2 c_{s}\right)^{\frac{p}{\alpha}}\left\|\left(u_{0}, g_{0}\right)\right\|_{D}^{\frac{p-1}{\alpha}}}\right\}$ with the data norm $\left\|\left(u_{0}, g_{0}\right)\right\|_{D}$ as in Theorem 1.2. Furthermore, the data-to-solution map $\left\{u_{0}, g_{0}\right\} \mapsto u$ is locally Lipschitz continuous. 
Theorem 5.1 can be proved similarly to Theorem 1.2, once the linear estimates of Theorem 1.1 are supplemented with an additional estimate in the new space $C^{\alpha}\left([0, T] ; L_{x}^{p}(0, \infty)\right)$. This task concerns both the pure linear IBVP (2.1) and the linear IVPs (3.1) and (3.16). In particular, the estimation of problem $(2.1)$ in the space $C^{\alpha}\left([0, T] ; L_{x}^{p}(0, \infty)\right)$ is straightforward. Decomposing $v=v_{1}+v_{2}$ as in the Proof of Theorem 2.1 and noting that $v_{1}$ and $v_{2}$ can be handled identically to each other, we use Minkowski's integral inequality to obtain

$$
\begin{aligned}
\left\|v_{1}(t)\right\|_{L_{x}^{p}(0, \infty)} & \lesssim \int_{k=0}^{\infty}\left(\int_{x=0}^{\infty}\left|e^{i a^{3} k x+i k^{2} t} k \widehat{g}\left(k^{2}\right)\right|^{p} d x\right)^{\frac{1}{p}} d k \\
& =\int_{k=0}^{\infty} k\left|\widehat{g}\left(k^{2}\right)\right|\left(\int_{x=0}^{\infty} e^{-\frac{1}{\sqrt{2}} p k x} d x\right)^{\frac{1}{p}} d k \simeq \int_{k=0}^{\infty} k^{1-\frac{1}{p}}\left|\widehat{g}\left(k^{2}\right)\right| d k .
\end{aligned}
$$

Thus, letting $k=\sqrt{\tau}$ and applying the Cauchy-Schwarz inequality in $\tau$, we find

$$
\left\|v_{1}(t)\right\|_{L_{x}^{p}(0, \infty)} \lesssim\left(\int_{\tau=0}^{\infty} \tau^{-\frac{1}{p}}\left(1+\tau^{2}\right)^{-\frac{2 s+1}{4}} d \tau\right)^{\frac{1}{2}}\|g\|_{H_{t}^{\frac{2 s+1}{4}}(\mathbb{R})} \simeq\|g\|_{H_{t}^{\frac{2 s+1}{4}}(\mathbb{R})}, \quad s>\frac{1}{2}-\frac{1}{p} .
$$

A similar estimate holds for $v_{2}$ and, overall, we deduce $\|v\|_{C^{\alpha}\left([0, T] ; L_{x}^{p}(0, \infty)\right)} \lesssim\|g\|_{H_{t}^{\frac{2 s+1}{4}}(\mathbb{R})}$.

The estimation of the linear IVPs (3.1) and (3.15) in the space $C^{\alpha}\left([0, T] ; L_{x}^{p}(\mathbb{R})\right)$ is also elementary. For $\frac{1}{2}-\frac{1}{p}<s<\frac{1}{2}$ and forcing of the form $F=\prod_{j=1}^{p} F_{j}$, we find

$$
\|U\|_{C^{\alpha}\left([0, T] ; L_{x}^{p}(\mathbb{R})\right)} \lesssim\left\|U_{0}\right\|_{H_{x}^{s}(\mathbb{R})}, \quad\|W\|_{C^{\alpha}\left([0, T] ; L_{x}^{p}(\mathbb{R})\right)} \lesssim T^{\alpha} \prod_{j=1}^{p}\left\|F_{j}\right\|_{C^{\alpha}\left([0, T] ; L_{x}^{p}(\mathbb{R})\right)} .
$$

Finally, the forced linear IVP estimates (3.17) and (3.18) are replaced by

$$
\sup _{t \in[0, T]}\|W(t)\|_{H_{x}^{s}(\mathbb{R})}+\sup _{x \in[0, \infty)}\|W(x)\|_{H_{t}^{\frac{2 s+1}{4}}(0, T)} \lesssim \sqrt{T} \prod_{j=1}^{p}\left\|F_{j}\right\|_{C^{\alpha}\left([0, T] ; L_{x}^{p}(\mathbb{R})\right)} .
$$

Combining all of the above estimates, we arrive at the analog of the linear Theorem 1.1 for $\frac{1}{2}-\frac{1}{p}<s<\frac{1}{2}$, which in turn implies the nonlinear Theorem 5.1 via a contraction mapping argument and the use of the well-known inequality $\left.|| v\right|^{p-1} v-|w|^{p-1} w\left|\lesssim\left(|v|^{p-1}+|w|^{p-1}\right)\right| v-w \mid$. Furthermore, following the above outline, one can also establish the finite interval version of Theorem 5.1, which provides the analog of Theorem 1.3 in the case of rough data $\frac{1}{2}-\frac{1}{p}<s<\frac{1}{2}$.

We conclude by noting that diffusion equations are typically studied in the literature via more classical techniques developed specifically for such equations with data in Lipschitz spaces (e.g., see Refs. 27, 1, 33, 18, and 32). However, as mentioned earlier, our objective here has been to demonstrate that the reaction-diffusion equation (1.1a) can be included in the theory originally developed for dispersive equations such as NLS, ${ }^{11} \mathrm{KdV},{ }^{12}$ and "good" Boussinesq, ${ }^{20}$ which relies on employing the unified transform of Fokas for deriving novel linear estimates along rays in the complex Fourier space.

\section{SUMMARY}

In this work, a novel approach that was recently developed for the analysis of nonlinear dispersive equations on domains with a boundary, such as the NLS and KdV equations on the half-line, has been advanced to study a reaction-diffusion equation with power nonlinearity both on the half-line and on a finite interval. The new approach exploits the linear solution formulae produced by Fokas's unified transform, a powerful method for the solution of linear evolution equations of any order, on a variety of physical domains and with any admissible boundary conditions. In this connection, the present work demonstrates that the novel unified transform approach is universal, in the sense that it can be used for analyzing a wide range of nonlinear evolution equations beyond dispersive ones, formulated not only on the half-line but also on a finite interval.

\section{ACKNOWLEDGMENTS}

The first author was partially supported by a grant from the Simons Foundation (No. 524469 to Alex Himonas).

\section{REFERENCES}

${ }^{1}$ N. Alikakos, "L $\mathrm{L}^{\mathrm{p}}$ bounds of solutions of reaction-diffusion equations," Commun. Partial Differ. Equations 4, 827-868 (1979).

${ }^{2}$ J. Bona, S. Sun, and B.-Y. Zhang, “A non-homogeneous boundary-value problem for the Korteweg-de Vries equation in a quarter plane,” Trans. Am. Math. Soc. 354, 427-490 (2002).

${ }^{3}$ J. Bona, S. Sun, and B.-Y. Zhang, "Nonhomogeneous boundary value problems of one-dimensional nonlinear Schrödinger equations,” J. Math. Pures Appl. 109, 1-66 (2018). 
${ }^{4}$ C. Budd, G. Collins, and V. Galaktionov, "An asymptotic and numerical description of self-similar blow-up in quasilinear parabolic equations," J. Comput. Appl. Math. 97, 51-80 (1998).

${ }^{\mathbf{5}}$ T. Cazenave, F. Dickstein, and F. Weissler, “On the structure of global solutions of the nonlinear heat equation in a ball," J. Math. Anal. Appl. 360, 537-547 (2009).

${ }^{6}$ J. Colliander and C. Kenig, "The generalized Korteweg-de Vries equation on the half-line," Commun. Partial Differ. Equations 27, 2187-2266 (2002).

${ }^{7}$ B. Deconinck, B. Pelloni, and N. Sheils, "Non-steady-state heat conduction in composite walls," Proc.: Math., Phys. Eng. Sci. 470, 20130605 (2014).

${ }^{8}$ Y. Egorov, V. Galaktionov, V. Kondratiev, and S. Pohozaev, "Global solutions of higher-order semilinear parabolic equations in the supercritical range," Adv. Differ. Equations 9, 1009-1038 (2004).

${ }^{9}$ A. Fokas, "A unified transform method for solving linear and certain nonlinear PDEs," Proc. R. Soc. London, Ser. A 453, 1411-1443 (1997).

${ }^{10}$ A. Fokas, A Unified Approach to Boundary Value Problems (SIAM, 2008).

${ }^{11}$ A. Fokas, A. Himonas, and D. Mantzavinos, “The nonlinear Schrödinger equation on the half-line," Trans. Am. Math. Soc. 369, 681-709 (2017).

${ }^{12}$ A. Fokas, A. Himonas, and D. Mantzavinos, "The Korteweg-de Vries equation on the half-line," Nonlinearity 29, 489-527 (2016).

${ }^{13}$ A. Fokas, A. Its, and L.-Y. Sung, "The nonlinear Schrödinger equation on the half-line," Nonlinearity 18, 1771-1822 (2005).

${ }^{14}$ A. Fokas and J. Lenells, "The unified method: I. Nonlinearizable problems on the half-line," J. Phys. A: Math. Theor. 45, 195201 (2012).

${ }^{15}$ A. Fokas and B. Pelloni, "A transform method for linear evolution PDEs on a finite interval," IMA J. Appl. Math. 70, 564-587 (2005).

${ }^{16}$ A. Fokas and E. Spence, "Synthesis, as opposed to separation, of variables," SIAM Rev. 54, 291-324 (2012).

${ }^{17}$ V. Galaktionov, "Incomplete self-similar blow-up in a semilinear fourth-order reaction-diffusion equation," Stud. Appl. Math. 124, 347-381 (2010).

${ }^{18}$ Y. Giga, “Solutions for semilinear parabolic equations in $\mathrm{L}^{\mathrm{p}}$ and regularity of weak solutions of the Navier-Stokes system,” J. Differ. Equations 62, 186-212 (1986).

${ }^{19}$ Y. Giga and R. Kohn, "Asymptotically self-similar blow-up of semilinear heat equations," Commun. Pure Appl. Math. 38, 297-319 (1985).

${ }^{20}$ A. Himonas and D. Mantzavinos, “The 'good' Boussinesq equation on the half-line," J. Differ. Equations 258, 3107-3160 (2015).

${ }^{21}$ J. Holmer, "The initial-boundary-value problem for the 1D nonlinear Schrödinger equation on the half-line," Differ. Integr. Equations 18, 647-668 (2005).

${ }^{22}$ J. Holmer, "The initial-boundary-value problem for the Korteweg-de Vries equation," Commun. Partial Differ. Equations 31, 1151-1190 (2006).

${ }^{23}$ B. Hu, Blow-Up Theories for Semilinear Parabolic Equations (Springer, 2018).

${ }^{24}$ C. Kenig, G. Ponce, and L. Vega, "Oscillatory integrals and regularity of dispersive equations," Indiana Univ. Math. J. 40, 33-69 (1991).

${ }^{25}$ J. Lenells and A. Fokas, “The unified method: II. NLS on the half-line with t-periodic boundary conditions," J. Phys. A: Math. Theor. 45, 195202 (2012).

${ }^{26}$ J. Lenells and A. Fokas, "The unified method: III. Nonlinearizable problems on the interval," J. Phys. A: Math. Theor. 45, 195203 (2012).

${ }^{27} \mathrm{~J}$. Lions and E. Magenes, Non-Homogeneous Boundary Value Problems and Applications (Springer-Verlag, 1972), Vol. 2.

${ }^{28} \mathrm{H}$. Matano and F. Merle, "Classification of type I and type II behaviors for a supercritical nonlinear heat equation," J. Funct. Anal. 256, 992-1064 (2009).

${ }^{29}$ B. Pelloni, "Well-posed boundary value problems for integrable evolution equations on a finite interval," Theor. Math. Phys. 133, $1598-1606$ (2002).

${ }^{30}$ B. Pelloni, "Well-posed boundary value problems for linear evolution equations on a finite interval," Math. Proc. Cambridge Philos. Soc. 136, 361-382 (2004).

${ }^{31}$ N. Sheils and D. Smith, "Heat equation on a network using the Fokas method," J. Phys. A: Math. Theor. 48, 335001 (2015).

${ }^{32}$ J. Smoller, Shock Waves and Reaction-Diffusion Equations, Grundlehren der Mathematischen Wissenschaften Vol. 258 (Springer-Verlag, 1994).

${ }^{33} \mathrm{~F}$. Weissler, "Local existence and nonexistence for semilinear parabolic equations in L p," Indiana Univ. Math. J. 29, 79-102 (1980). 